\title{
Identification and classification of cis-regulatory elements in the amphipod crustacean Parhyale
}

\section{hawaiensis}

Dennis A Sun ${ }^{1}$ and Nipam H Patel ${ }^{2,3^{*}}$

1. Department of Molecular and Cell Biology, University of California, Berkeley

2. Marine Biological Laboratory, Woods Hole, MA

3. Department of Organismal Biology and Anatomy, University of Chicago, Chicago, USA

${ }^{*}$ corresponding author

\section{Primary Findings}

- Omni-ATAC-Seq identifies cis-regulatory elements genome-wide during crustacean embryogenesis

- Combined short- and long-read RNA-Seq improves the Parhyale genome annotation

- ImpulseDE2 analysis identifies dynamically regulated candidate regulatory elements

- NucleoATAC and HINT-ATAC enable inference of nucleosome occupancy and transcription factor binding

- Fuzzy clustering reveals peaks with distinct accessibility and chromatin dynamics

- Integration of accessibility and gene expression reveals possible enhancers and repressors

- Omni-ATAC can identify known and novel regulatory elements

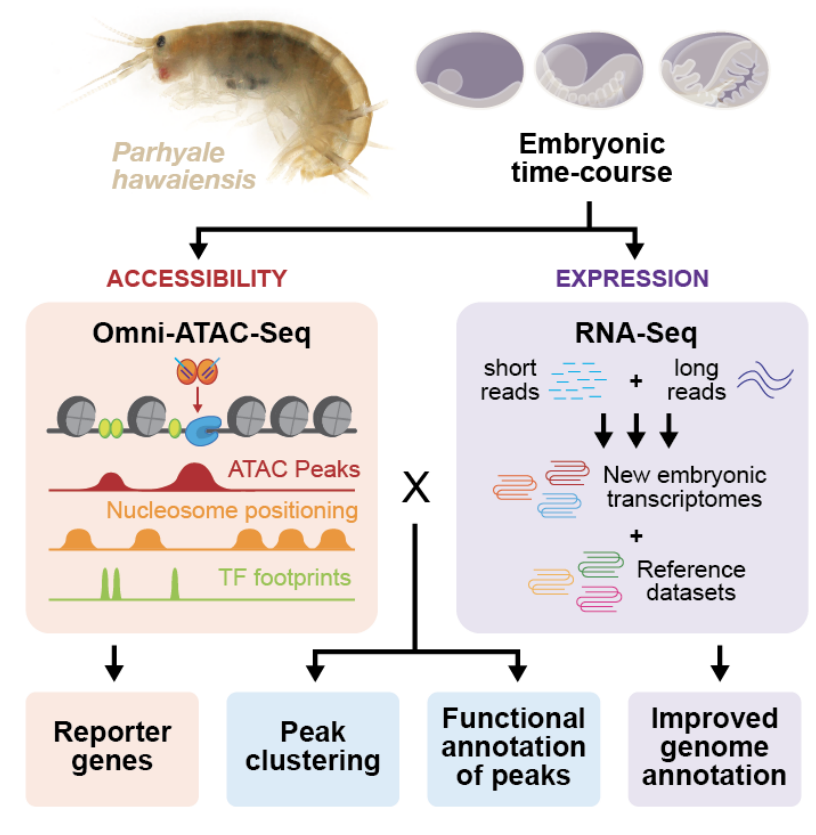

\begin{abstract}
Emerging research organisms enable the study of biology that cannot be addressed using classical "model" organisms. The development of novel data resources can accelerate research in such animals. Here, we present new functional genomic resources for the amphipod crustacean Parhyale hawaiensis, facilitating the exploration of gene regulatory evolution using this emerging research organism. We use Omni-ATAC-Seq, an improved form of the Assay for Transposase-Accessible Chromatin coupled with next-generation sequencing (ATAC-Seq), to identify accessible chromatin genome-wide across a broad time course of Parhyale embryonic development. This time course encompasses many major morphological events, including segmentation, body regionalization, gut morphogenesis, and limb development. In addition, we use short- and long-read RNA-Seq to generate an improved Parhyale genome annotation, enabling deeper classification of identified regulatory elements. We leverage a variety of bioinformatic tools to discover differential accessibility, predict nucleosome positioning, infer transcription factor binding, cluster peaks based on accessibility dynamics, classify biological functions, and correlate gene expression with accessibility. Using a Minos transposase reporter system, we demonstrate the potential to identify novel regulatory elements using this approach, including distal regulatory elements. This work provides a platform for the identification of novel developmental regulatory elements in Parhyale, and offers a framework for performing such experiments in other emerging research organisms.
\end{abstract}




\section{Introduction}

Advances in genomic techniques have facilitated genetic research in emerging research organisms. As sequencing tools become more sophisticated and less expensive over time, understanding what genes an organism expresses at any time in development becomes more straightforward. The expansion of genome sequencing has accelerated to such a degree that cost per gigabase of genome sequence has decreased at a rate faster than predicted by Moore's Law (Wetterstramd). Moreover, the establishment of techniques including RNAi and CRISPR-Cas9 mutagenesis has made genetic ablation available to researchers working in a range of different research organisms, enabling the rapid characterization of gene function. RNAi knockdown has been applied to various insects (MITO et al., 2011; Schmitt-Engel et al., 2015), chelicerates (Sharma et al., 2013), flatworms (Rouhana et al., 2013; Srivastava et al., 2014), and numerous other organisms to study gene function. More recently, CRISPR-Cas9 mutagenesis has enabled both targeted ablation and targeted transgenesis in organisms as diverse as cephalopods (Crawford et al., 2020) and reptiles (Rasys et al., 2019). Using these tools, it is now possible to understand how genes and genomes evolve across the tree of life.

While changes to gene function have been shown to play some role in evolutionary processes, changes to gene regulation are thought to be a more important force in shaping the diversity of cell types, morphologies, and populations across clades (Mattioli et al., 2020; Signor and Nuzhdin, 2018; Wittkopp et al., 2004). The evolution of gene regulation is governed by changes in the function of two gene-regulatory modes: changes to trans-regulatory factors and changes to cis-regulatory elements. Trans-regulatory factors may include transcription factors, noncoding RNAs, and other diffusible elements that modify the expression of distant genes, while cis-regulatory elements are considered regions of the genome that locally mediate gene regulation.

The identification and characterization of possible trans-regulatory factors in a genome can be accomplished with relative ease using genome sequencing. For example, functional annotation of transcriptomes is able to easily identify transcription factors based on highly conserved protein domains (Ofran et al., 2007; Wang and Brown, 2006). Many bioinformatic tools also exist to identify microRNAs and long noncoding RNAs through sequence-based approaches (Duan et al., 2020; Liu et al., 2014). RNA-Seq approaches can also enable the identification of differentially expressed transcripts which might be important for biological function (Costa-Silva et al., 2017). Thus, it is possible to identify many of the possible trans-regulatory factors within an organism's genome with straightforward RNA sequencing approaches.

Cis-regulatory elements (CREs), on the other hand, have historically proven difficult to identify, owing to their rapid evolution over short evolutionary distances. Unlike protein-coding sequences, which can be identified through mRNA sequencing and predictable open reading frames, cis-regulatory elements are composed of complex assemblies of transcription factor binding sites, which can be computationally challenging to identify (Li et al., 2015; Wasserman and Sandelin, 2004). These sites can change dramatically over short periods of time; for example, in the context of the even-skipped stripe 2 enhancer across several Drosophila species, the position of critical transcription factors binding sites shifted in as short a span as 1-2 million years divergence (Ludwig et al., 1998). Such rapid evolutionary flexibility, while important for driving short- and long-term evolutionary processes, makes the identification of such elements in novel research organisms a considerable challenge.

In recent years, novel techniques for identifying CREs have been developed, which rely on the fact that CREs often occur in regions of accessible chromatin. The earliest, including DNAse I hypersensitivity and Formaldehyde-Assisted Isolation of Regulatory Elements (FAIRE-Seq) enabled researchers to locate accessible chromatin regions and discover novel regulatory elements in a range of research organisms (Giresi et al., 2007; Lai et al., 2018; Pérez-Zamorano et al., 2017). However, such techniques were hampered by low signal-to-noise ratios, the requirement for very large amounts of tissue, 
and technically challenging protocols. In 2013, Buenrostro et al. published papers describing the Assay for Transposase-Accessible Chromatin with next-generation sequencing (ATAC-Seq), a novel technique using a hyperactive version of the Tn5 transposase, as a way of identifying accessible chromatin regions genome-wide (Buenrostro et al., 2015). In this paper, they demonstrated that ATAC-Seq performed comparably to previous accessibility techniques with reduced tissue requirements and increased convenience and speed.

ATAC-Seq has proved to be an effective technique for traditional model systems, and has offered a true revolution for emerging model systems. The low material input requirements of ATAC-Seq are of particular use in emerging research organisms, for which generating the millions of cells required for previous techniques would prove challenging. Researchers have applied ATAC-Seq to gar embryos in order to study the evolution of limb development (JL and Shubin, 2015) and regenerating acoel flatworms and hydra to study regeneration (Cazet et al., 2021; Gehrke et al., 2019), among numerous other examples. Thus, ATAC-Seq is now established as a generalizable tool that is particularly well-suited to accelerating work in emerging research organisms.

In 2017, Corces et al. reported a further-improved version of ATAC-Seq, Omni-ATAC-Seq, which provides several improvements to the standard ATAC-Seq protocol (Corces et al., 2017). In particular, it has been reported to have a greater signal-to-noise ratio, decreased mitochondrial read contamination, and applicability to fixed and frozen tissues, with only minor modifications to the buffers and detergents used in the standard ATAC-Seq protocol.

In this paper, we apply the Omni-ATAC-Seq protocol to embryos of the amphipod crustacean Parhyale hawaiensis, an emerging research organism for the study of arthropod development, evolution, and regeneration (Paris et al., 2021; Stamataki and Pavlopoulos, 2016; Sun and Patel, 2019). We identify dynamic regions of open chromatin ("peaks") across a broad swath of Parhyale developmental time. We comprehensively analyze our Omni-ATAC-Seq data to predict the position of nucleosomes along the genome and infer the footprints of transcription factors bound to peaks. Using fuzzy clustering (Kumar and E Futschik, 2007), we partition our peaks into groups based on similar accessibility trajectories, revealing groups of peaks with different transcription factor footprint enrichment and nucleosome occupancy that are active at different points in development. In addition, we use short- and long-read RNA-Seq to improve the Parhyale genome annotation and investigate the relationship between accessibility and gene expression over time during development.

Parhyale has served as a platform for foundational discoveries about such processes as body plan evolution (Martin et al., 2015), the evolution of arthropod limbs (Bruce and Patel, 2020), and the evolution of regeneration (Konstantinides and Averof, 2014). By facilitating the identification of regulatory elements in this research organism, our work will enable other researchers to investigate the complexities of gene regulatory evolution in these processes and others. Furthermore, by enabling the assessment of cis-regulatory elements in Parhyale, we open up avenues to investigate fundamental mechanisms of gene regulation in the understudied non-insect crustacean clade.

While this work primarily focuses on Parhyale, our methods can provide examples of how one can perform thorough analyses of ATAC-Seq and RNA-Seq data using existing tools to generate hypotheses about gene expression dynamics and regulatory element function. Such approaches can be applied to a diverse range of organisms, and will facilitate deeper understanding of gene regulation across the tree of life. 


\section{Results}

\section{Omni-ATAC-Seq identifies open chromatin across Parhyale developmental stages}

To identify developmental regulatory elements, we performed Omni-ATAC-Seq on 15 stages of Parhyale embryonic development (Fig. 1). For each developmental stage, we generated two Omni-ATAC-Seq libraries using five embryos each, and fixed and DAPI stained sibling embryos to confirm staging (Fig. 1A). The developmental stages catalogued in our dataset are illustrated in Fig. 1B, and representative fixed, DAPI-stained sibling embryos are illustrated in Fig. 1C. Additional brightfield images of embryos prior to tagmentation can be found in Supp. Fig. $1 \mathrm{~A}$.

To assess the quality of our libraries, we employed a battery of standard tests used to assess the quality of ATAC-Seq data (Supp Fig. 1A). Omni-ATAC-Seq libraries were also compared to previous ATAC-Seq libraries (see Supplementary Data), and had greater library size, higher numbers of non-duplicated reads, and lower amounts of mitochondrial DNA contamination (Supp. Fig. 2B, C). Fragment size analysis of Omni-ATAC-Seq reads after mapping revealed distinct 1-nucleosome and 2-nucleosome peaks, indicating that tagmentation was efficient (Supp Fig. 1E, F).

To determine whether our Omni-ATAC-Seq data represented genuine enrichment in accessible chromatin regions, we examined promoter accessibility genome-wide. A hallmark of successful ATAC-Seq experiments is strong enrichment of reads in gene promoters (Yan et al., 2020). We evaluated our ATAC-Seq using the most recent phaw_5.0 gene annotations (obtained from Leo Blondel, hereafter referred to as the "Blondel annotation") at the start of annotated mRNAs and across mRNA lengths, and compared our results to RNA-Seq read pileups (Fig. 2A-B). Omni-ATAC-Seq signal is enriched symmetrically at mRNA starts, whereas RNA-Seq reads are enriched 3' of mRNA starts. In addition, Omni-ATAC-Seq data shows greater enrichment at promoters than over annotated mRNA regions, and decreased enrichment outside of annotated mRNAs. These data suggest that our Omni-ATAC-Seq performed as expected in identifying promoter regions genome-wide.

To assess whether our libraries were capable of capturing significant variation over time, we performed Principal Component Analysis (PCA) on our libraries (Fig. 2C). PCA analysis revealed that our libraries were primarily separated along two principal components, PC1 (60\% of variation) and PC2 (18\% of variation), with a considerable drop in variation explained in other PCs (Supp. Fig. 2D). PC1 appeared to be associated with developmental time, with earlier developmental stage libraries showing a negative loading, and later developmental stages showing a positive loading.

To identify regions of dynamically accessible chromatin, we used the ImpulseDE2 (IDE2) pipeline (Fischer et al., 2018). ImpulseDE2 differs from other software for differential expression analysis in that it allows the investigation of trajectories of dynamic expression over large numbers of timepoints. Identifying differential expression is difficult for more categorical differential expression software such as edgeR and DESeq2, which use pairwise comparisons between timepoints to assess change over time (Love et al., 2014; Robinson et al., 2010). In addition, IDE2 enables the detection of transient changes in gene expression or accessibility during a time course.

We used Genrich (Gaspar) to call significant peaks $(q<0.05)$ in each of our 15 timepoints independently, and merged overlapping peaks across timepoints using bedtools (Quinlan and Hall, 2010), yielding 190,078 genomic regions which we used as our "peaks" in downstream analyses. The dynamic accessibility of these peaks is illustrated in a heatmap in Fig. 2D. Of these peaks, 163,227 (85.87\%) were classified as having statistically significant variation (padj $<0.01$ ) over our time-course. Of all peaks, 60,909 (37.32\%) were classified as having transient expression dynamics and 88,231 (54.05\%) were classified as showing monotonic expression dynamics (strictly up or down over the time-course). These results indicate that we were able to identify many dynamically accessible regions across the Parhyale genome. 
IDE2 produces a fitted model of accessibility defined as the product of two sigmoid functions for each of the peaks used in the analysis (Fischer et al., 2018). We used these model fits to summarize the global properties of the dynamic peaks we identified. First, we estimated the number of models that achieved their maximum accessibility during each of the 15 timepoints sampled (Fig. 2F). The timepoints during which models most frequently showed their highest accessibility were $S 13, S 21$, and $S 27$, representing approximately the earliest, middle, and latest timepoints.

We then used these IDE2 model fits to understand how the principal components of our libraries were related to accessibility dynamics (Supp. Fig. 2G). We extracted the 100 peaks with the most positive and negative loadings in PC1 and PC2 and plotted their IDE2 model fits. Based on these plots, we inferred that PC1 captured variation along developmental time, with positively-loaded peaks showing a "low early, high late" accessibility trajectory, and negatively-loaded peaks showing a "high early, low late" accessibility trajectory. Peaks with high positive loading in PC2 appeared to be those with highest accessibility in the middle developmental stages.

These global analysis results indicate that our Omni-ATAC-Seq experiments captured information normally found in ATAC-Seq data, including strong enrichment at promoters. Overall, the low mitochondrial read contamination, large library size, and low fraction of duplicated reads suggest that our Omni-ATAC-Seq data are of high quality. In addition, the results of the ImpulseDE2 differential accessibility analyses indicate that the vast majority of accessible regions in the Parhyale genome show dynamic accessibility over developmental time.

\section{Improving the Parhyale genome annotation using short- and long-read RNA-Sequencing}

While Omni-ATAC-Seq signal showed enrichment across annotated mRNA starts in a genome-wide analysis, careful examination of individual genes and gene models indicates that many of the Blondel gene annotations are fragmented. For example, among the Parhyale Hox genes, 7 of 9 the genes showed discrepancies in annotation compared to previous RACE data (Serano et al., 2016), with $l a b, p b, U b x$, and $a b d-A$ showing fragmented gene models (Supp. Fig. 3C, D).

A more accurate genome annotation would improve both genome-wide analyses and enable the precise classification of candidate regulatory elements as promoters, exonic and intronic regulatory elements, and intergenic regulatory elements. To generate a more complete genome annotation, we performed RNA-Seq using two approaches: short-read sequencing using the Illumina NovaSeq platform and long-read sequencing using Oxford Nanopore technology. For four developmental stages (S13, S19, S21, and S23), we generated triplicate RNA-Seq libraries, which were sequenced using a short-read approach (Illumina NovaSeq) and a long-read approach (Oxford Nanopore) (Fig. 3A, C; Supp. Fig. 1B).

We assembled multiple transcriptomes from each of the sequencing approaches (Fig. 3B). Using the short-read sequences, we generated transcriptomes using the Trinity RNA-Seq pipeline (Haas et al., 2013), including a combined de novo transcriptome using all four developmental stages, stage-specific de novo transcriptomes, and a genome-guided transcriptome. We observed using BUSCO that the merged transcriptomes containing reads from all four developmental stages (Trinity-all, Trinity-GG) had reduced BUSCO scores compared to stage-specific transcriptomes (Trinity-S13 to Trinity-S23)(Fig 3D). One possible explanation for this could be the difficulty of assembling reads with high levels of heterozygosity, as has been previously described of the Parhyale genome (Kao et al., 2016). For long-read sequencing, we mapped the reads to the phaw_5.0 genome using minimap2 and assembled a transcriptome using StringTie2 (Kovaka et al., 2019). We also used StringTie2 to generate a transcriptome containing both short- and long-reads. While the long-read transcriptome (StringTie2 L) showed a low BUSCO score, the short + long read transcriptome (StringTie2 SL) showed comparable BUSCO to our other transcriptomes (Fig. 3D).

To generate a more complete genome annotation, we used the Mikado pipeline to merge our transcriptomes with previously-generated transcriptomes, including from the Kao et al. 2016 manuscript 
(also generated using Mikado) and from Heather Bruce (unpublished, generated using Trinity). The Mikado pipeline and others (e.g. EvidentialGene) takes advantage of the previously observed variable ability of transcriptome assemblers to produce complete gene models for different types of genes (Gilbert, 2019; Venturini et al., 2018). We evaluated the Mikado merged transcriptome using BUSCO, and observed a comparable score to the best of the individual transcriptomes ( $90.9 \%$ complete), and a marked improvement as compared to the Blondel annotation ( $80.9 \%$ complete).

We first examined the models in the merged Mikado transcriptome for the Parhyale Hox cluster compared to RACE data and the Blondel annotation (Supp. Fig. 3 C-E). The Mikado merged transcriptome showed an improvement relative to the Blondel models overall, with complete models for $p b, H o x 3, D f d$ and Scr. While the Mikado pipeline was only able to recover one transcript isoform each for the three posterior Hox genes, for abd-A and $U b x$, the isoforms identified were more complete than in the Blondel annotation. Moreover, the Mikado combined transcriptome was able to produce a more complete gene model for Hox3 than the previous RACE data or Blondel annotation. Combining the RACE data with our Mikado transcriptome, we have assembled the most complete annotation of the Parhyale Hox cluster to date.

To quantify the level of fragmentation of gene models in our dataset, we generated a series of manual gene annotations based on additional previous sequence information generated using RACE. Among 143 previously-identified RACE transcripts, we selected 49 multi-exonic transcripts that appeared to have a single promoter based on the Omni-ATAC-Seq data (Fig. 3E). For each of these RACE transcripts, we manually annotated the extent of the first and last exon by comparing RNA-Seq read pileups to RACE data and the current genome annotation. We also identified the Omni-ATAC-Seq peak most likely to capture the gene promoter, based on the strength of the peak (as evaluated by the number of timepoints over which we observed a statistically significant peak), as well as overlap to RNA-Seq read data. We used these manual annotations (see Supplementary Data) to evaluate the gene models from each of the different transcript sources (transcriptomes or gene annotations).

For each of the 49 genes in our dataset, we evaluated whether any models in each of the transcript sources in our dataset overlapped the promoter peak (Fig. 3F). Among the transcript sources, the Trinity de novo transcriptomes had the highest number of genes where at least one model overlapped with the promoter peak. However, the Trinity models we observed often contained numerous spurious and fragmented transcripts. Among the remaining transcript sources, the Mikado merged transcriptome had the highest number of genes where at least one model overlapped with the promoter peak (82\%).

In addition, we assessed the degree of fragmentation of gene models across the 49 RACE transcripts (Fig 4A). For each gene, we determined first whether any single gene model spanned the first and last exon, representing a "complete" transcript. If there was not a single model, we next assessed whether two separate models overlapped with the first and last exon, or if all models only overlapped either the first or last exon. These results together formed a measure of transcript completeness.

Comparing the different transcript sources, the StringTie2 SL transcriptome had the highest number of "complete" transcripts, with the Mikado merged transcriptome having a slightly lower number of "complete" transcripts. Given that the Mikado merged transcriptome likely captured transcripts from more diverse sources - including previous 454 transcriptomes and adult tissue transcriptomes that were used to generate the Kao, Blondel, and Bruce datasets - we performed further downstream analysis using the Mikado merged transcriptome as our reference.

To evaluate the quality of the Mikado merged transcriptome as a reference, we compared its performance to the Blondel annotation in a variety of metrics (Fig. 3G). The Mikado transcriptome produced a larger number of total gene models than are found in the Blondel annotation. Using bedtools, we attempted to assign a candidate promoter peak to each transcript in each genome annotation, and observed that the Mikado transcriptome had a larger number of transcripts with an assigned promoter, further suggesting genome-wide gene completeness. 
To further assess the quality of the two genome annotations, we performed automated functional annotations to assign gene names and functions. We used two approaches: eggNOG and OrthoFinder (Emms and Kelly, 2019; Huerta-Cepas et al., 2019). eggNOG is a rapid and lightweight genome annotation software, and assigns gene names, KEGG pathway information, and GO terms, among numerous other metrics, to gene models. Trinotate is a more comprehensive transcript annotation pipeline, while OrthoFinder facilitates the identification of orthogroups between provided peptide libraries.

We observed that the Mikado transcriptome included a greater number of GO-term assigned genes, eggNOG named genes, and eggNOG uniquely-named genes than found in the Blondel annotation (Fig. 3G). We used OrthoFinder to assign orthogroups between the Mikado and Blondel transcriptomes and the list of all Drosophila melanogaster peptides from the UNIPROT-SWISSPROT database. The Mikado and Blondel transcriptomes produced similar numbers of orthogroups as analyzed by OrthoFinder; however, the Mikado transcriptome produced a greater number of 1:1 orthogroups, which proved useful for downstream GO term enrichment analysis.

We further examined the quality of the Mikado and Blondel transcriptomes by evaluating Omni-ATAC-Seq signal and RNA-Seq signal genome-wide at mRNA starts (Fig. 3H-I). We observed a comparable RNA-Seq signal, but a higher Omni-ATAC-Seq signal for the Mikado transcriptome as compared to the Blondel annotation, suggesting a stronger enrichment of promoters in our dataset. These results together suggest that the Mikado transcriptome produces a more complete genome annotation, capturing more of the bona fide genes in the Parhyale genome than previously available.

Using the Mikado transcriptome as a reference, we assigned Omni-ATAC-Seq peaks to a number of different spatial categories along the genome (Fig. 4F, see Methods). We observed that intergenic peaks make up the vast majority $(71.3 \%)$ of Omni-ATAC peaks. We further partitioned the intergenic peaks into proximal and distal segments, with distal intergenic peaks representing those peaks $>10 \mathrm{~kb}$ away from the nearest gene. The majority of peaks in our dataset (51\%) were distal intergenic peaks, indicating that much of the Parhyale regulatory landscape is composed of distal regulatory elements. Nearly a fifth of regulatory elements $(18.2 \%)$ were also located on contigs that did not contain genes, and were classified as "unknown".

As our peaks represent the merged combination of peaks across individual timepoints, we also evaluated the proportion of peaks from each time-point belonging to each spatial category (Fig. 4F). Early developmental stages appeared to have a greater proportion of peaks in "unknown" regions, while later developmental stages appeared to have an increased number of peaks in intergenic regions, with the number of promoter, exonic, and intronic peaks remaining similar over time.

\section{Inferring nucleosome positioning and transcription factor footprints from Omni-ATAC-Seq}

ATAC-Seq and other accessibility approaches primarily identify regions of open chromatin in the genome. However, with deep enough sequencing, one can infer additional information from the pattern of insertions of ATAC-Seq adapters into the genome. Using NucleoATAC, we predicted the positions of nucleosomes near Omni-ATAC-Seq peaks over time. In addition, using HINT-ATAC, we inferred the footprints of transcription factors bound to accessible peaks over time.

NucleoATAC enables the prediction of nucleosome positioning from ATAC-Seq data (Schep et al., 2015). We applied NucleoATAC to a window of +/-500bp around our Omni-ATAC-Seq peaks at each developmental stage. NucleoATAC depends on a stereotypical signal of Tn5 insertion around nucleosomes referred to as a "V-plot", which resembles the fragmentation pattern around chromatin subjected to chemical fragmentation. We observed across developmental time-points a clear V-plot signal generated by NucleoATAC (example of V-plot from S21 libraries shown in Supp. Fig. 4A).

A well-established signal of nucleosome positioning found across animals is the presence of strong +1 and -1 nucleosome positioning around gene promoters, and a depletion of nucleosomes at RNA-Polll binding sites (referred to as nucleosome-free region, or NFR) (Brogaard et al., 2012; 
Radman-Livaja and Rando, 2010). We visualized NucleoATAC signal and nucleosome occupancy around mRNA starts genome-wide, and observed strong signals associated with +1 and -1 nucleosomes (Fig. 4 A, B; Supp. Fig. 4 B-D). We also observed a depletion of nucleosomes and a decrease in NucleoATAC signal between the +1 and -1 nucleosomes, which may represent nucleosome-free regions. This signal was observed consistently in each of our stage-specific libraries (Supp. Fig. 4 E, F). These data indicate that we were able to identify the stereotypical signal of nucleosomes at promoters from our Omni-ATAC data. Moreover, these results suggest that NucleoATAC is more generally able to infer nucleosome positions and nucleosome-free regions within our dataset genome-wide .

In addition to inferring nucleosome positions, deeply sequenced ATAC-Seq data also enables the inference of transcription factor footprints. We used HINT-ATAC to identify transcription factor footprints in Omni-ATAC-Seq peaks from each developmental stage library (Li et al., 2019). In addition, we used HINT-ATAC to evaluate the enrichment of TF footprints across developmental time. We observed that HINT-ATAC enriched TF footprints appeared to group into three broad clusters based on the timing of highest enrichment: early, S21/S22, and late (Fig. 4D, E). These trends were true both when examining all JASPAR CORE PWMs, and when examining only those JASPAR CORE PWMs from Drosophila. These results are consistent with the general trends observed in our Omni-ATAC-Seq data, which suggest that the early, middle, and late timepoints in development have the greatest variation.

The data generated from these analyses enable the visualization of chromatin accessibility, predicted nucleosome position, inferred transcription factor binding, and RNA-Seq expression at individual loci across around half of Parhyale development. The depth of this data is illustrated in Fig 4C at the predicted promoter of the Parhyale Engrailed-1 locus for stage S21. Thus, for any genomic region of interest, one can develop a comprehensive prediction of the local chromatin environment at each of the 15 timepoints in our dataset.

\section{Identifying gene regulatory programs from Omni-ATAC-Seq using fuzzy clustering}

To assess the potential for our dataset to provide new biological insights, we attempted to identify distinct gene regulatory programs based on differential accessibility. We used the Mfuzz package to perform fuzzy c-means clustering on the matrix of read counts generated from our Omni-ATAC-Seq peaks (Kumar and E Futschik, 2007). Fuzzy clustering differs from categorical clustering approaches such as hierarchical clustering and k-means clustering in that it assigns a probability for each element in the dataset to fall into each of the identified clusters. For example, rather than a given peak falling into either cluster A or B, a given peak might have a $95 \%$ chance of falling into cluster $A$ and a $5 \%$ chance of calling into cluster $\mathrm{B}$.

The Mfuzz package allows the specification of two parameters: $c$, or the number of clusters to create from the data, and $m$, the stringency of the clustering. To determine the optimal number of clusters in our fuzzy c-means clustering, we varied the c-value from 3 to 13, and evaluated the quality of clustering using the cluster overlap metric provided by Mfuzz, as well as the silhouette score metric (Supp. Fig. 4)(Kumar and E Futschik, 2007). We determined that 9 was the number of clusters that allowed for the highest mean silhouette score while avoiding clusters below the silhouette score average and minimizing size variability between clusters. Figure $5 \mathrm{~A}$ illustrates the top 10,000 most significant peaks called by ImpulseDE2 using t-SNE, where each point is colored by its IDE2 model max fit, while Figure 5B illustrates the same clusters, colored by their Mfuzz cluster.

We evaluated the differences between clusters through a variety of approaches. Figure 6D shows the standardized raw accessibility scores of peaks in each cluster over time. We first assessed the spatial category distribution of each cluster compared to the global average. Clusters 1, 2, and 8 appeared to be enriched for "unknown" regions, while clusters 4, 5, 6, 7, and 9 appeared to be enriched for distal intergenic regions relative to the global distribution. In addition, we used the IDE2 model fits for each cluster to evaluate the general accessibility dynamics of each cluster (Fig. 5E, F). Clusters 1 and 2 appear 
to be enriched for peaks that achieve high accessibility early in development and decrease in accessibility over time. Clusters 3, 4, 5, and 6 appear to be enriched for peaks that achieve maximum accessibility at different timepoints along developmental time. Cluster 7 appears to be enriched for peaks that show low accessibility early in development, and increased accessibility late in development. Finally, clusters 8 and 9 showed a more striking pattern, with Cluster 8 composed of peaks that appear to have a pulsatile increase in accessibility around stages S21-S22, and Cluster 9 composed of peaks with an opposite pattern: those with a sudden pulsatile decrease around S21-S22. Thus, the 9 clusters identified in our analysis appeared to have distinct patterns of accessibility over time.

We further investigated the clusters identified by Mfuzz using additional metrics in order to understand the biological significance of these clusters. First, we investigated the relationship between accessibility dynamics and nucleosome positioning between clusters (Fig. 5G). For each developmental time point, we visualized Omni-ATAC accessibility (dashed line, black axis) compared to NucleoATAC signal (dotted line, blue axis) and NucleoATAC nucleosome occupancy (solid line, pink axis) for peaks belonging to each cluster. We observed that peaks belonging to clusters $3,4,5,6,7$, and 9 all appeared to have an inverse relationship between accessibility and NucleoATAC signal. Moreover, these peaks all appeared to have a depletion of nucleosome occupancy at peak centers relative to the region around peaks. By contrast, peaks in clusters 1, 2, and 8 appeared to show a counterintuitive and opposite result: both high NucleoATAC signal and high nucleosome occupancy at timepoints during which those peaks achieved maximum accessibility.

To assess the biological function of each of the 9 Mfuzz clusters, we performed GO term enrichment analysis. To assign functions to each peak, we extracted the Drosophila melanogaster gene assigned to the nearest gene for each peak, and performed GO enrichment analysis on the list of unique gene names associated with peaks from each cluster relative to the list of all OrthoFinder-assigned gene names in the genome. Fig. 6A illustrates the top 10 most-enriched GO terms for each of the clusters. While we were not able to identify significantly enriched GO-terms (FDR < 0.05 ) for Clusters 1,2 , and 8 , Clusters 3, 4, 5, 6, 7, and 9 all showed enrichment of developmental GO-terms. These enriched GO-terms generally appeared to match the expected biological functions of peaks based on their accessibility dynamics. For example, Cluster 3 peaks, which generally achieve maximum accessibility during germ band elongation stages in development, showed enrichment for GO terms including head segmentation and neuroblast fate, two processes which take place during this developmental period. Meanwhile, Cluster 7 peaks appeared to be enriched for neuronal terms (neuropeptide signaling pathway, synaptic target recognition, neuron recognition), potentially reflecting the function of genes near these peaks which achieve their maximum accessibility towards the very end of the developmental time-course, during which much of the morphology of the organism has been specified, and the embryo begins to twitch inside the egg.

To further investigate the clusters in our dataset, we examined the enrichment of TF footprints generated from HINT-ATAC in each of our clusters. For each cluster, we compiled all of the TF footprints predicted across developmental stages for all peaks within that cluster. We compared the enrichment of those TF footprints to our randomly generated background data, and examined all JASPAR CORE transcription factors, as well as the subset of JASPAR CORE transcription factors found in Drosophila.

We observed strong differential enrichment of different groups of transcription factors in each of our 9 clusters. Notably, particular families of transcription factors appeared enriched in several of the clusters. For example, Cluster 1 and Cluster 2 appeared to have enrichment for footprints matching $\mathrm{C} 2 \mathrm{H} 2$ zinc-finger transcription factors and homeodomain transcription factors. Clusters 4 and 7 showed enrichment for footprints matching beta helix-loop-helix (bHLH) transcription factors. Meanwhile, clusters 5,8 , and 9 showed strong enrichment for footprints of ethylene response factor (ERF), DM-type intertwined zinc-finger factor (DM IZF), and forkhead domain (FKH) transcription factor families, respectively. 
Given that many JASPAR position weight matrices come from non-arthropod sources, we attempted to identify possible Drosophila motif matches for the enriched transcription factor footprints found in the JASPAR CORE dataset by comparing the most-enriched JASPAR CORE and JASPAR Drosophila PWMs. We used the STAMP tool through the JASPAR webpage to align motif sequences based on similarity (Mahony and Benos, 2007). We identified groups of aligned PWMs between non-Drosophila and Drosophila PWMs (see Supp. Fig. 6). Selected pairs of motifs are shown in Fig. 6C for each cluster. For some pairs, we were able to identify candidate Drosophila transcription factors that appeared highly similar to non-Drosophila sequences. For example, in Cluster 9, we observed strong enrichment for FKH family transcription factors, whose PWMs matched the br(var.4) and slp1 PWMs. This suggests that peaks found in Cluster 9 may be regulated by br(var.4) and slp1 binding in Parhyale.

For other clusters, we had variable success. For example, we were unable to identify a strong Drosophila match to explain the strong enrichment of ERF transcription factors (found in Arabidopsis thaliana) in Cluster 5. Nonetheless, footprints matching ERF transcription factors appeared to be highly enriched in Cluster 5, suggesting that there may be a transcription factor within the Parhyale genome whose position weight matrix matches that of ERF transcription factors, and which is important for the regulation of Cluster 5 peaks.

Altogether, our results indicate that clustering Omni-ATAC data using accessibility can identify groups of transcription factors with similar accessibility trajectories. In addition, these clusters can be further analyzed using nucleosome occupancy, GO enrichment, and transcription factor footprint enrichment to understand possible biological functions and genetic mechanisms behind differential accessibility.

\section{Concordant and discordant expression and accessibility dynamics appear across development}

To understand the relationship between peak accessibility and gene expression, we examined the correlation between Omni-ATAC accessibility in individual peaks with respect to RNA-Seq expression for the nearest gene at each of the developmental stages for which we generated RNA-Seq data. We first examined the relationship between accessibility at all peaks compared to their nearest genes (Fig. 8A) and observed a very weak (Pearson $R^{2}=0.11-0.15$, Spearman $\left.\rho=0.1-0.14\right)$ but statistically significant $(p$ $<0.001$ ) positive correlation between peak accessibility and the expression of nearby genes. When we examined only promoter peaks (Fig. 8B), we observed a slightly greater correlation between accessibility and gene expression (Pearson $R^{2}=0.19-0.22$, Spearman $\rho=0.17-0.25$ ) correlation that was also significant $(p<0.001)$. Notably, at each developmental stage, we observed a higher correlation between accessibility and expression at promoter peaks than at all peaks.

We expect that both enhancers and repressors might be found among our ATAC-Seq peaks. To further classify peaks, we examined the relationship between the change in accessibility of individual peaks and the change in expression of nearby genes. We used DESeq2 to evaluate the log fold change of both expression (RNA-Seq) and accessibility (ATAC-Seq) for all genes and peaks. We then correlated the differential accessibility and gene expression for all peak - gene pairs where both the differential accessibility of a peak and expression of the peak's nearest gene were statistically significant (padj < 0.05). When examining all peaks, we observed a weak but significant correlation between log fold change in accessibility and gene expression (Fig. 8C). This correlation was slightly stronger at promoter peaks (Fig. 8D).

Among our differential accessibility and expression analyses, we classified two broad classes of peak-gene pairs representing concordant and discordant accessibility and expression dynamics. Peaks with concordant accessibility and gene expression were those where the sign of the log fold change in expression and accessibility were in agreement - for example, peaks where an increase in accessibility was observed concurrently with an increase in expression. Meanwhile, peaks with discordant accessibility and gene expression were those where an increase in accessibility was observed concurrently with a 
decrease in expression, or vice versa. We observed both classes of peak-gene pairs among all peaks, but also among promoter peaks. The classification of peaks as concordant or discordant in accessibility and gene expression may provide insight both into the function of individual peaks as enhancers or silencers, and the presence of repressive regulation within promoter peaks.

\section{Omni-ATAC-Seq re-identifies known Parhyale regulatory elements}

To assess the practical usefulness of our Omni-ATAC-Seq dataset, we compared our Omni-ATAC-Seq peaks to known regulatory elements in Parhyale. A very limited set of regulatory elements have been described in Parhyale: a muscle reporter, PhMS (Pavlopoulos and Averof, 2005); a heat shock element, HS2a (Pavlopoulos et al., 2009); an embryonic ubiquitous reporter, PEB (Danielle Liubicich, unpublished); and two reporter constructs for Parhyale Opsin-1 and Opsin-2 (Ramos et al., 2019). We were unable to locate any Omni-ATAC-Seq peaks for the Parhyale Opsin genes, likely owing to their late expression (outside of our developmental time course) and very low cell number (a handful of cells per eye). However, strong Omni-ATAC-Seq enrichment was observed at the PhMS, HS2a, and PEB elements, as illustrated in Supp. Fig. 7.

Each of these reporter constructs consists of the first exon and intron of a gene. In each case, we observed an Omni-ATAC peak overlapping the 5' end of the gene. For the PhMS reporter (Fig. 7B), previous work had identified a cluster of putative b-HLH transcription factor binding sites. These sites were captured within our Omni-ATAC peak. The HS2a reporter has been reported to contain two binding sites for heat shock factor (HSF) proteins upstream of a minimal promoter (PhHsp70), both of which are captured within our Omni-ATAC peak (Fig. 7D). Finally, the PEB element appears to contain two Omni-ATAC peaks. These results suggest Omni-ATAC is able to identify the position of functional cis-regulatory elements, and has been able to capture those regions important for cis-regulatory element function.

\section{Minos transposase reporter assays can reveal novel promoters and distal enhancers}

Given that our Omni-ATAC peaks were able to capture known regulatory elements, we attempted to use these peaks to identify novel regulatory elements. Careful examination of developmentally important genes revealed that many are surrounded by large numbers of peaks $(>10)$ spread over large genomic distances. To further filter our Omni-ATAC peaks, we identified regions of sequence conservation to another amphipod crustacean, Hyalella azteca (Poynton et al., 2018), using the VISTA sequence alignment software (Ratnere and Dubchak, 2009)(Fig. 8A). Hyalella serves as a useful comparison to Parhyale, as its genome size is considerably smaller ( $240 \mathrm{Mb})$, but as it is also an amphipod crustacean, we expect that key developmental regulatory elements might have some level of sequence conservation.

Among our Omni-ATAC peaks, we took two strategies to identify candidate reporter elements. In the first approach, we identified all peaks within $5 \mathrm{~kb}$ of mRNA starts (Fig. 10E). In doing so, we were able to identify a handful of genomic regions in which we were able to locate both a putative promoter and a candidate proximal enhancer. We also identified several putative promoters, which we tested in isolation. In the second approach, we examined the genomic regions around important developmental genes of interest. Many of the genes we examined showed very large numbers of strong peaks, and were therefore intractable to thorough analysis. For the purposes of this study, we focused on three regions: the region around the Engrailed-1 gene, the region around the $S p-69$ gene, and the region around the Heat shock protein 70 complex, where two previous cis-regulatory elements (PhMS and HS2a) had been identified.

To assess the function of candidate regulatory elements identified by Omni-ATAC-Seq, we employed a Minos transposase reporter assay (Fig. 8G). In this assay, we injected Minos transposase mRNA along with a transposon donor plasmid containing a reporter gene construct that expressed DsRed 
to 1- and 2-cell embryos. Once per day, from 3-10dpf, we screened for DsRed expression on a Zeiss LSM780 confocal microscope.

The reporters we examined are summarized in Table 1. In the course of our experiments, we observed numerous spontaneous expression patterns (Supp. Fig. 5). These expression patterns may represent rare integration events, or may reflect fortuitous insertions into regions of the genome with promiscuous regulatory sequences that facilitate expression.

One expression pattern observed from the Sp69-p3 enhancer appeared to be repeated in 5 embryos along the $\sim 300$ we injected. This expression pattern was characterized by strong expression in the head, antenna 1, and antenna 2 (Supp. Fig. 6). In the same set of experiments, we also observed three spontaneous expression patterns that appeared to differ amongst each other, labeling different regions of the limbs and gills. It is possible that the repeated expression observed from the Sp69-p3 enhancer represents a genuine expression pattern. We tested this hypothesis by raising three Sp69-p3 head-expressing animals to adulthood and mating the two adults that showed the strongest expression during development. However, none of the offspring of this cross showed obvious head expression.

Among the novel reporter constructs we tested, two showed robust expression: pMi(Hsc70-4) and $\mathrm{pMi}(\mathrm{ne} 1)-\mathrm{Hsp} 70-\mathrm{p} 2$. The pMi(Hsc70-4) construct was constructed using the putative promoter peak of the Hsc70-4 gene (Fig. 10K, M). This reporter showed strong expression in a cluster of neurons associated with the eye beginning at around $9 \mathrm{dpf}$ (Fig. 10L). The expression pattern of these neurons appears to be distinct from those observed using a synthetic 3XP3 enhancer, which has previously been used as a positive marker of transformation Parhyale (Pavlopoulos and Averof, 2005). Thus, it is possible to build new reporters from our Omni-ATAC peaks using putative promoter peaks.

The second reporter that showed robust expression was the pMi(ne1)-Hsp70-p2 construct, which contains the PhHsp70 minimal promoter along with a strong peak extracted from the Hsp70 cluster, about $30 \mathrm{~kb}$ away from the location of the minimal promoter (Fig. 10H, J). Hsp70-p2 drives strong expression in cells of the embryonic yolk (Fig. 10I). Some yolk cells labeled by this reporter moved dynamically around the yolk, and while others appeared static and showed spongiform morphology (Supp. Video 1). We observed defects in the development of the dorsal portion of the embryo in mid-stage ( $6-7 \mathrm{dpf})$ embryos injected with this construct, which stereotypically displayed a separation between the embryo proper and the eggshell (Fig. 10l, bottom panel, marked with arrow). While we were unable to establish a genetic line using this construct, we observed strong and reproducible expression in many embryos. This reporter is the first indication that distal enhancers exist and can be identified in Parhyale.

While it remains challenging to identify novel cis-regulatory elements in Parhyale, our results suggest that functional regulatory elements can be identified from our Omni-ATAC data. Among the numerous peaks identified in our dataset, we expect that many will reveal novel gene regulatory dynamics. Future work to identify such elements will require the optimization of reporter expression and transgenic strategies to increase the throughput and sensitivity of reporter gene assays.

\section{Discussion}

New genomic data enable deeper understanding of underlying biology. This is especially true in emerging research organisms, for which data and resources are limited. This work provides a wealth of genomic resources for the amphipod crustacean Parhyale hawaiensis. In addition to providing Omni-ATAC-Seq and RNA-Seq data for a broad developmental time course, our work generates multiple new transcriptomes, an updated genome annotation, a catalog of dynamically accessible chromatin regions, and predictions of nucleosome occupancy and transcription factor binding. Moreover, our thorough analysis of regulatory element dynamics through clustering, GO enrichment, predicted TF binding, and correlations with RNA-Seq present numerous hypotheses for cis-regulatory element function. Such data will support researchers in the growing Parhyale community in efforts to identify and 
characterize developmental regulatory elements, and provide the foundations for more advanced approaches, including single-cell sequencing techniques, which rely on high-quality reference datasets.

\section{The Parhyale genome contains many distant and dynamic regulatory elements}

From our data, we were able to glean new information about the global dynamics of gene regulation during Parhyale development, as well as the composition of the Parhyale genome. We observed that the vast majority of our peaks showed dynamic accessibility over developmental time, and that peaks could have a variety of different temporal dynamics, including transient increases and decreases in accessibility alongside more absolute increases and decreases. These results indicate that much of the Parhyale genome undergoes dynamic changes in accessibility over developmental time.

With an improved genome annotation, we were also able to determine that the vast majority $(>70 \%)$ of regulatory elements in Parhyale are located in intergenic regions, and that among all elements, a majority ( $51 \%)$ were located greater than $10 \mathrm{~kb}$ away from the nearest gene. These results are notable, given that many of the currently studied protostome genomes are small (for example, Drosophila melanogaster is $180 \mathrm{Mb}$, Tribolium castaneum is $200 \mathrm{Mb}$, and Bombyx mori is $530 \mathrm{Mb}$ ). In such organisms, regulatory elements tend to occur within short distances from promoters, and it is common in such systems to attempt to build reporter genes using a short window upstream of the promoter of a gene of interest.

Parhyale is an example of an arthropod with a large genome $(3.6 \mathrm{~Gb}$, or $\sim 10 \%$ larger than Homo sapiens at $3.2 \mathrm{~Gb}$ ). Given the large genome size, and the large proportion of elements located distant to genes, we expect that regulatory elements within Parhyale may be located more distantly from gene promoters than in other arthropods. Future attempts at building reporter genes in this organism will need to account for the large number of distant regulatory elements, which may be critical to proper reporter expression.

Our data are among the first to identify regulatory elements genome-wide in a non-insect arthropod (Gatzmann et al., 2018; Kissane et al., 2021), and also examine one of the largest sequenced arthropod genomes available to date (Kao et al., 2016). These data will enable other researchers to examine the relationship between genome size and regulatory element composition across more diverse taxa.

\section{Combined short- and long-read transcriptomes can improve genome annotations}

In addition to providing a resource for the Parhyale community, our work can also serve as a guide for other researchers working on developing such datasets in emerging research organisms. First, our work demonstrates the utility of combining short- and long-read sequencing in generating more accurate genome annotations for emerging research organisms. Even with a small number of sequences ( 1.2 million reads) generated using Nanopore sequencing, we were able to generate a transcriptome with a moderate BUSCO score $(71 \%)$. Combining short reads and long reads (StringTie2 SL), we were able to generate a transcriptome with both a high BUSCO score $(91.8 \%)$ and low rate of gene fragmentation.

Moreover, the completeness of the StringTie2 SL transcriptome met or exceeded that of several of our Trinity-derived transcriptomes. Examination of individual genomic regions in the Parhyale genome indicates that Trinity generates numerous spurious transcripts, which can be confounding for whole-genome analysis. Thus, for other researchers with access to a genome assembly, we would strongly recommend performing both short- and long-read sequencing, and assembling using multiple different assembly strategies to generate higher quality genome annotations. For those without access to a high-quality genome, we would caution against relying strictly on transcripts generated from software such as Trinity, which in our system generated many transcripts that did not appear to match with previous RACE data. Filtering transcripts by expression values, as well as employing 
transcriptome-merging pipelines such as Mikado (in the case of a high-quality genome) and EvidentialGene (in the absence of a genome), would likely help remove such spurious transcripts.

\section{Deep analysis of ATAC-Seq data can reveal more than just dynamic accessibility}

While the most direct analysis of ATAC-Seq data can enable the identification of dynamic regulatory elements, our work uses additional tools to infer nucleosome positioning and transcription factor binding from our ATAC-Seq data. Using NucleoATAC, we were able to recover clear signals of nucleosome positioning at promoters, a quality observed in numerous organisms. Using HINT-ATAC, we were able to predict transcription factor binding across developmental stages. Such analyses enable a glimpse of the possible chromatin landscape of the genome, particularly in the absence of more direct and specific, but also more time-consuming and expensive approaches such as ChIP-Seq or CUT\&RUN.

In addition to performing inference, we were able to identify clusters of peaks with potentially distinct biological functions using fuzzy clustering. These clusters of peaks appeared to differ in their accessibility dynamics, nucleosome positioning, and enrichment of transcription factor footprints, indicating that meaningful biological differences can be gleaned from deeper analysis of ATAC-Seq data. For example, we observed strong enrichment in Cluster 9 for FKH transcription factors footprints matching the PWMs for Drosophila br(var.4) and slp1. The strongest peaks in this cluster appeared to share a common decrease in accessibility during stages S21-S22, and an enrichment for genes with GO terms related to cytoskeletal function. This group of peaks appeared to show a decrease in accessibility at a time point during which the embryo undergoes a dramatic morphogenetic event in which it splits along the midline. Based on our data, it is possible that FKH containing transcription factors may play a role in that important morphogenetic event.

\section{Omni-ATAC-Seq identifies old and new regulatory elements, including enhancers}

Our data were able to capture most of the previously-identified regulatory elements that had been described for Parhyale. In addition, we were able to demonstrate that our data contain new regulatory elements. Notably, we demonstrate the first identification of a distal regulatory element, Hsp70-p2, located about $30 \mathrm{~kb}$ from the PhHsp70 minimal promoter. These results are the first identification of an enhancer separated by a large distance from a minimal promoter element in this organism.

Our results demonstrate that Omni-ATAC-Seq is able to identify novel regulatory elements, and we expect that numerous new reporter genes will be built from these data. Previous work relied on examining candidate regulatory elements within individual genomic regions, or attempting to build new reporters through random integration events. With this dataset, it is now possible to identify candidate regulatory elements for any gene of interest and to screen for expression patterns using the Minos reporter system. As this approach enables the direct investigation of candidate regulatory regions with known sequence, this will also enable the generation of novel synthetic reporters generated by modifying endogenous sequences. Such future directions will enable thorough dissection of regulatory elements to determine how transcription factor binding influences gene expression during Parhyale development.

While we were unable to identify regulatory elements that drove clear expression patterns for the handful of genes we tested, numerous additional peaks near these genes remain to be investigated. Future work to build new reporters in Parhyale may require the development of more efficient transgenesis and screening strategies. For example, improving the efficiency of CRISPR-mediated homologous recombination or CRISPR-mediated NHEJ transgenesis could enable researchers to insert a reporter near a candidate regulatory region of interest. Moreover, future approaches will also need to account for the numerous distant regulatory elements in the Parhyale genome, which may be important for gene regulation. Examining local DNA interactions using approaches such as $\mathrm{Hi}-\mathrm{C}$ or other chromatin conformation capture approaches will be instrumental in identifying distant regulatory regions. 
Together, these approaches have enabled the exploration of chromatin dynamics and transcription factor binding in an emerging model organism. Our work illustrates how a single, easily adapted protocol can yield data amenable to deep analysis. We would recommend that other researchers using ATAC-Seq or similar assays in emerging model organisms also take advantage of the additional information that could be gleaned from deep analysis of their data, which could provide further insights into how both local and global changes to nucleosome occupancy and transcription factor binding influence their biological processes of interest.

\section{Conclusion}

By combining Omni-ATAC-Seq with RNA-Seq across a broad developmental time course, our work is able to identify and classify numerous candidate cis-regulatory elements in the genome of the amphipod crustacean Parhyale hawaiensis. We demonstrate how deep analysis of Omni-ATAC data can facilitate the identification of peaks with distinct accessibility, nucleosome occupancy, and transcription factor footprint enrichment. We further classify peaks as concordant or discordant regulatory elements by integrating differential accessibility and differential expression, revealing candidate activating and repressive elements found in distal and proximal regulatory elements. Moreover, we show the potential to identify novel reporter genes using candidate promoters and enhancers from our data.

This work provides a substantial resource to the Parhyale community, and should accelerate the study of gene regulation in this emerging research organism. In addition, our work can serve as a framework for other researchers deploying ATAC-Seq and RNA-Seq approaches in emerging research organisms. Through deep analysis of ATAC-Seq data, combined short- and long-read sequencing, and integration of accessibility and gene expression, such researchers can identify regulatory elements with distinct biological functions and advance the study of gene regulation in their organisms of interest. 
bioRxiv preprint doi: https://doi.org/10.1101/2021.09.16.460328; this version posted September 18, 2021. The copyright holder for this preprint (which was not certified by peer review) is the author/funder, who has granted bioRxiv a license to display the preprint in perpetuity. It is made A Collect early Duplicatteil|\$blpapłesaCC-BY-NC-ND 4.0 International license.
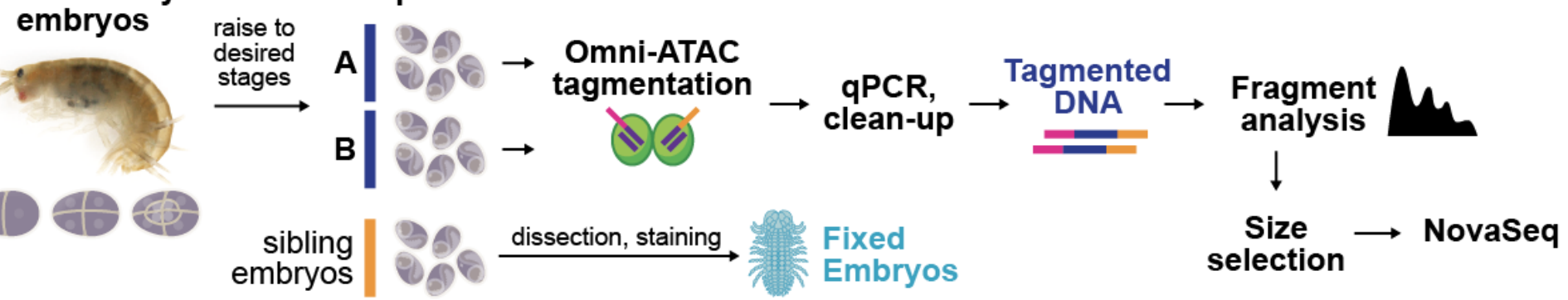

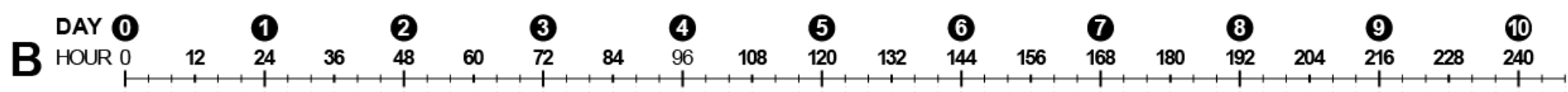

germband elongation

limb bud formation

limb morphogenesis

digestive ceca elongation
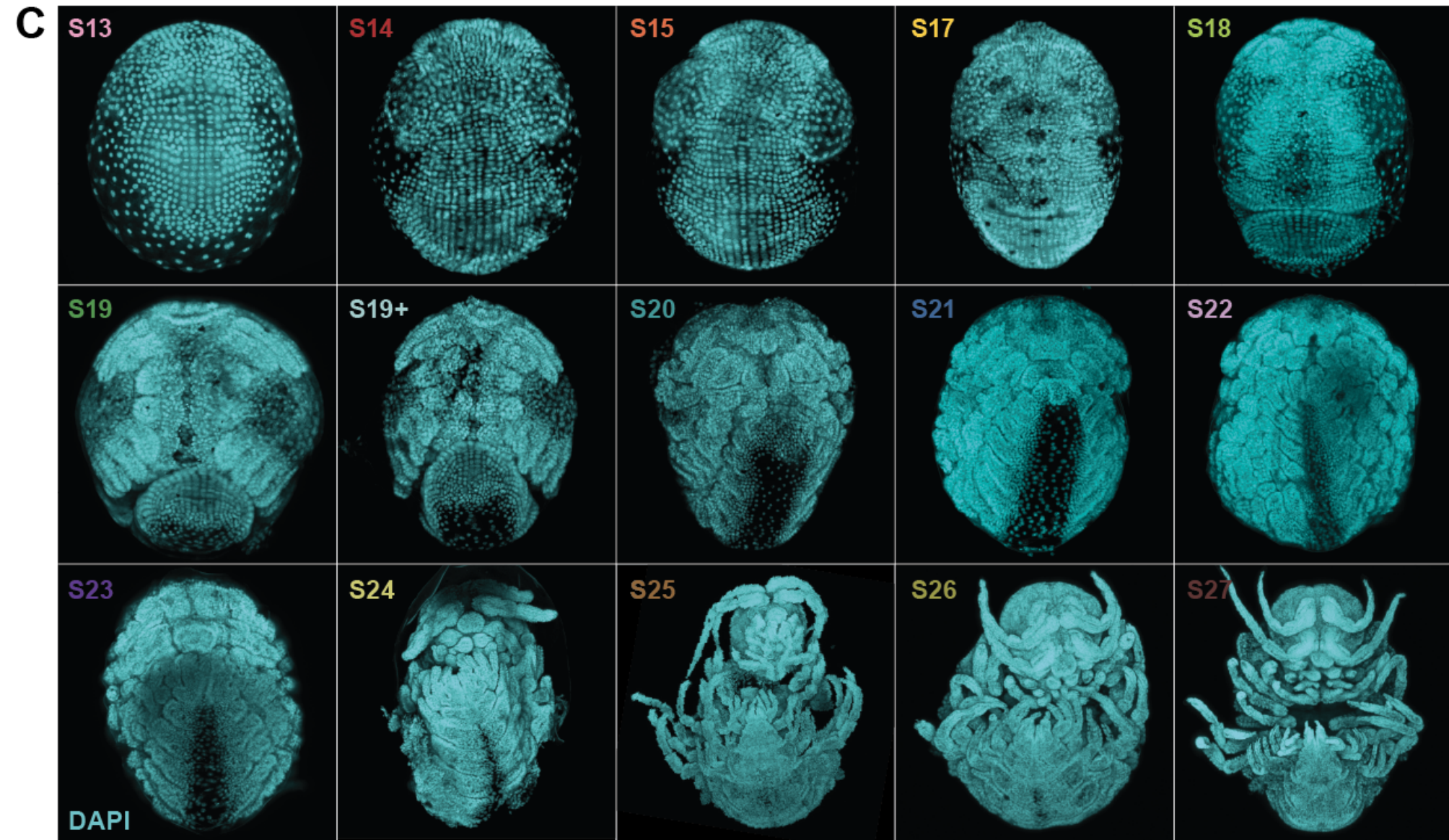

DAPI
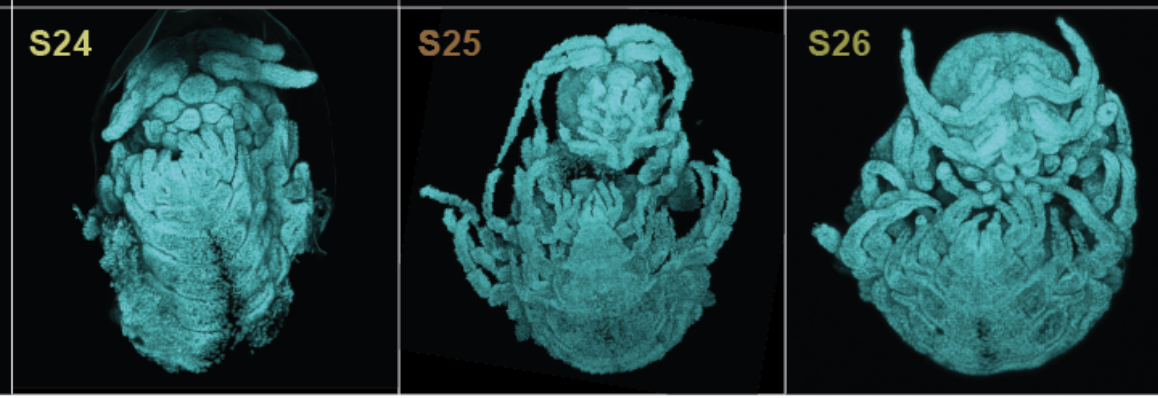
bioRxiv preprint doi: https://doi.org/10.1101/2021.09.16.460328; this version posted September 18, 2021. The copyright holder for this preprint (which was not certified by peer review) is the author/funder, who has granted bioRxiv a license to display the preprint in perpetuity. It is made available under aCC-BY-NC-ND 4.0 International license.

\section{Fig. 1: Time-course ATAC-Seq in Parhyale hawaiensis embryos.}

A) Overview of ATAC-Seq protocol. Embryos were collected at early developmental stages and raised to specific developmental timepoints. Duplicate libraries were generated using 5 embryos each from a single clutch of sibling embryos.

B) Timeline of developmental stages. RNA-Seq libraries were also generated for timepoints marked with a star.

C) Representative embryo images from clutches used for Omni-ATAC-Seq. Embryos are stained with DAPI and mounted ventral-side up. 
bioRxiv preprint doi: https://doi.org/10.1101/2021.09.16.460328; this version posted September 18, 2021. The copyright holder for this preprint (which was not certified by peer review) is the author/funder, who has geented bioBxiv a license to display the preprint in perpetuity. It is made

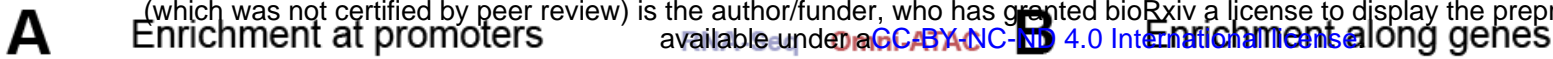
RNA-Seq Omni-ATAC

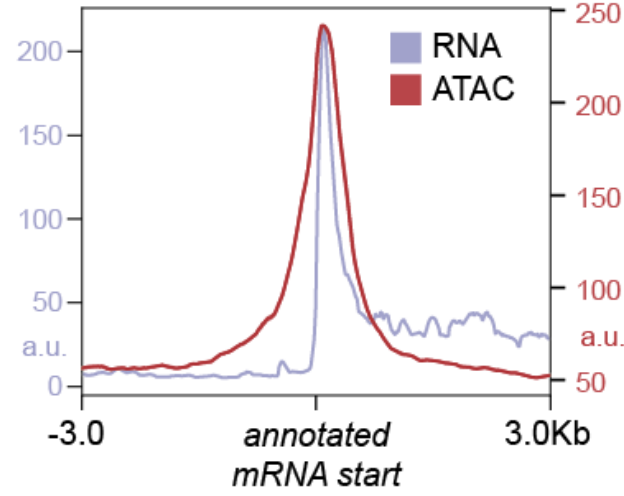

C PCA: Top 1000 most variant peaks

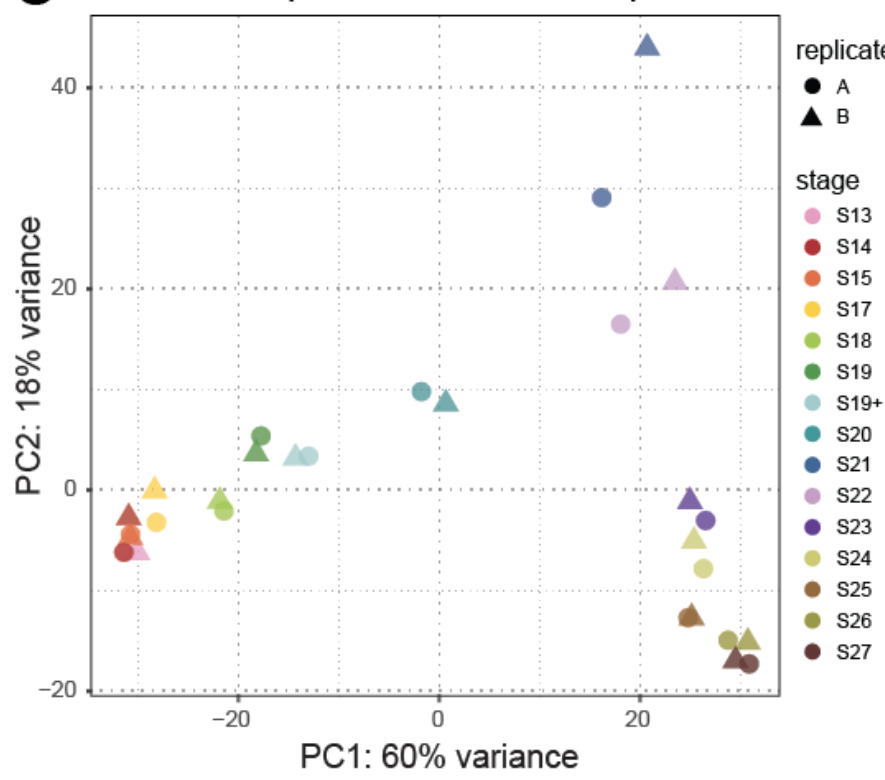

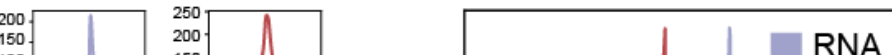

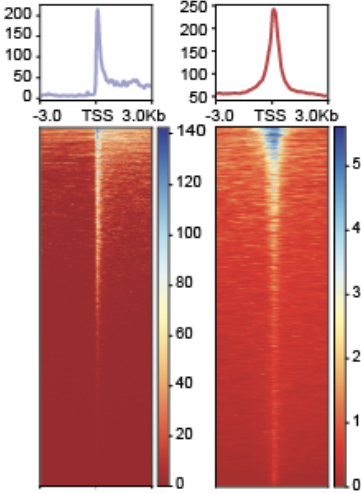

D Omni-ATAC peaks

론

휸

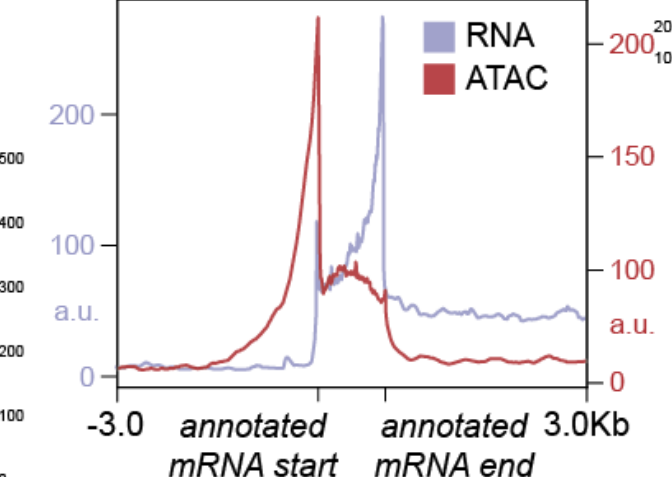

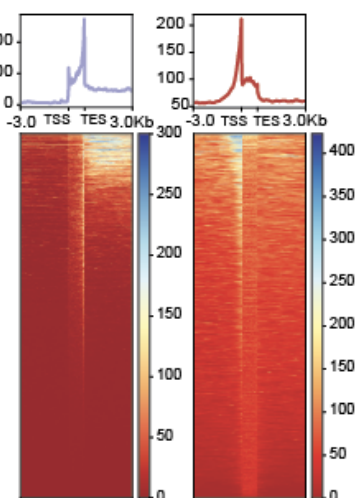

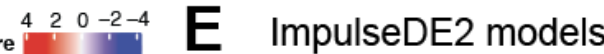

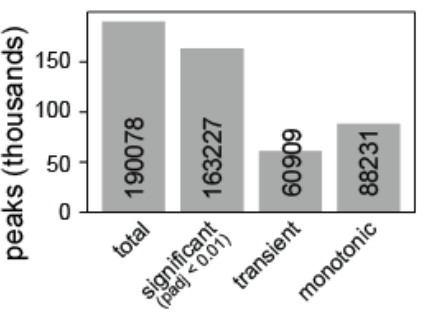

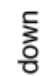

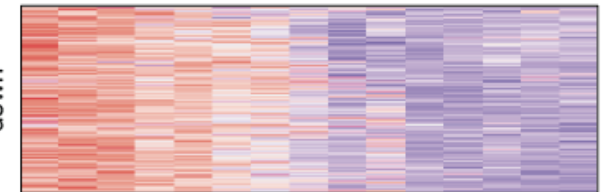

윽

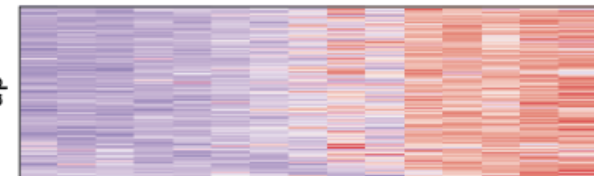

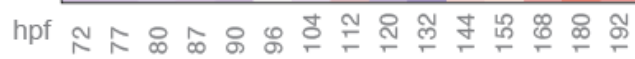

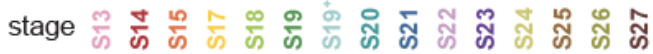

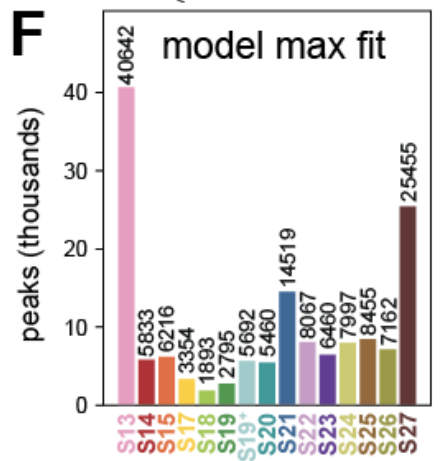


Fig. 2: Whole-genome analysis of ATAC-Seq reveals enrichment of promoter signals, differentially accessible regions
A) Omni-ATAC and RNA-Seq signal enrichment at promoters. Annotated mRNA start positions are aligned, and average signal across genomic positions is plotted for either ATAC-Seq or RNA-Seq signal.
B) Omni-ATAC and RNA-Seq signal enrichment over gene bodies.
C) PCA of Omni-ATAC libraries generated using DESeq2. PCA loadings were assigned to the top 1000 variant peaks, where variance was measured as row variance across all libraries.
D) Heatmap of Omni-ATAC accessibility dynamics, generated from ImpulseDE2 model fits.
E) Bar chart indicating number of peaks classified as significant, transient, and monotonic by ImpulseDE2.
F) Bar chart quantifying stage of maximum accessibility for significant ImpulseDE2 model fits. 
bioRxiv preprint doi: https://doi.org/10.1101/2021.09.16.460328; this version posted September 18, 2021. The copyright holder for this preprint (which was not certified by peer review) is the author/funder, who has granted bioRxiv a license to display the preprint in perpetuity. It is made

A Collect early embryos

Triplicate libibraries

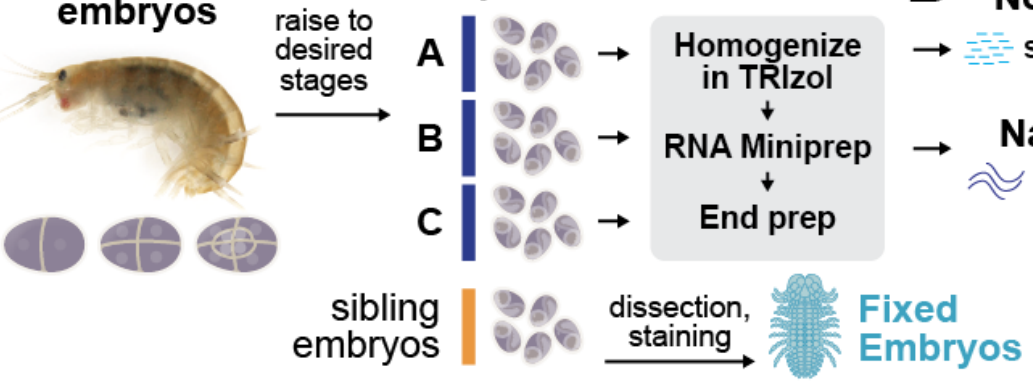

C
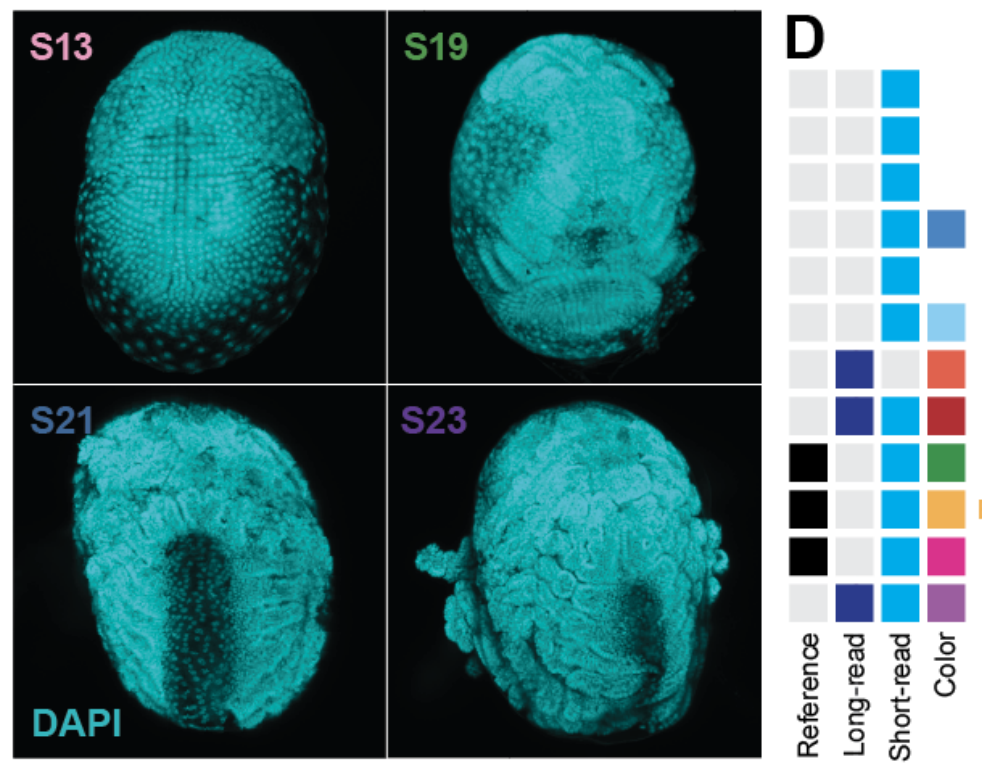

NovaSeq - Trinity $\rightarrow$ short read

short reads $\left.-\begin{array}{c}\text { Trinity } \\ \text { RNA-Seq } \rightarrow \text { transcriptomes }\end{array}\right]$

Nanopore

long read,

Mikado

long reads $\stackrel{\text { Stringtie2 } \rightarrow \text { transcriptomes }}{\text { longte }}$

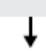

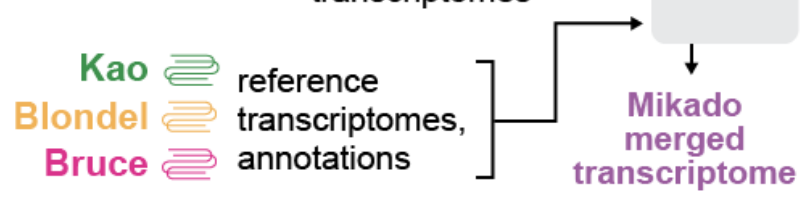

\section{BUSCO Assessment}

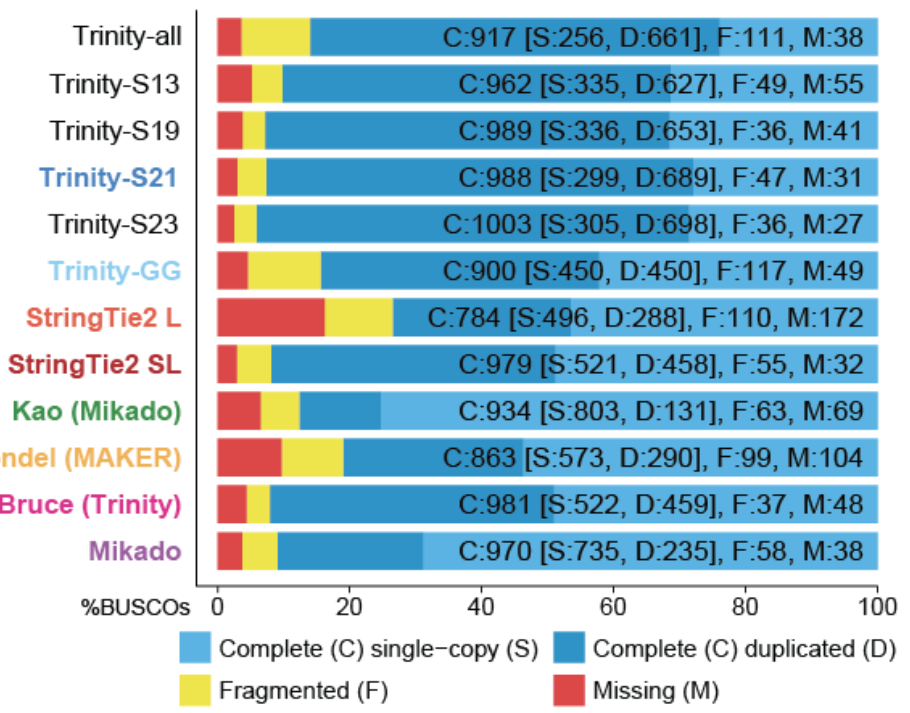

E

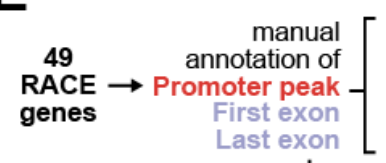

transcriptomes
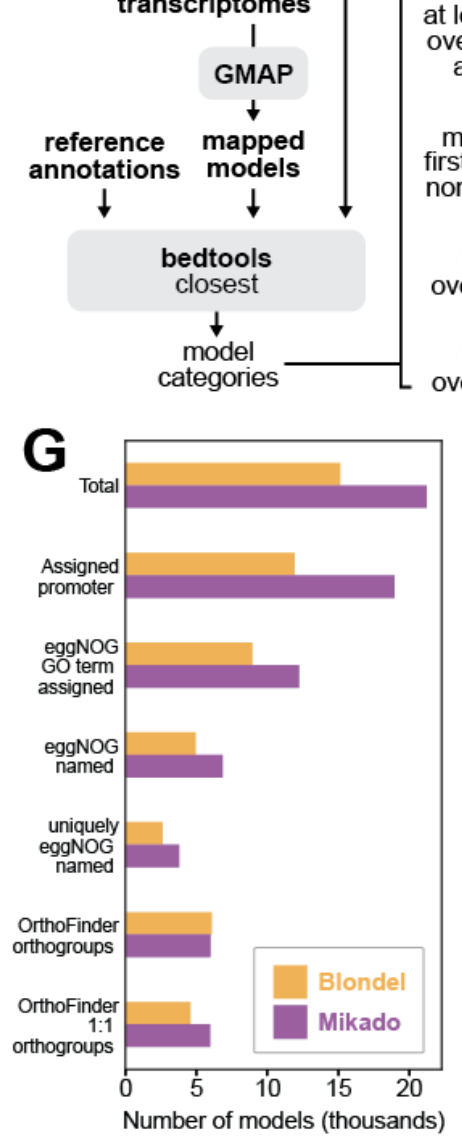
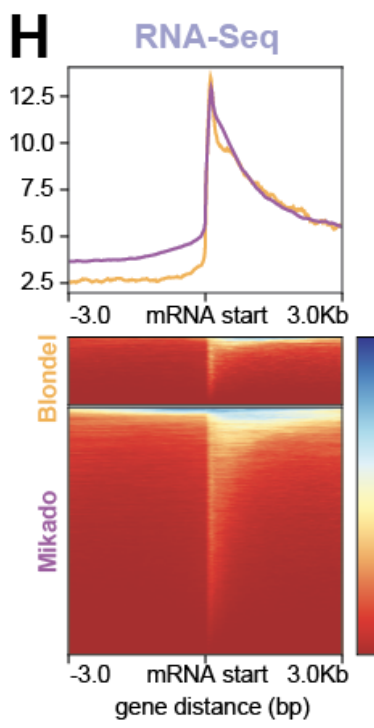

Promoter peak

Genrich peaks

Omni-ATAC

RNA-Seq

Single

at least one model overlaps both first and last exon

Split

models overlap first/ last exon, but none overlap both

\section{First only
Models only} overlap first exon

Last only

Models only

overlap last exon

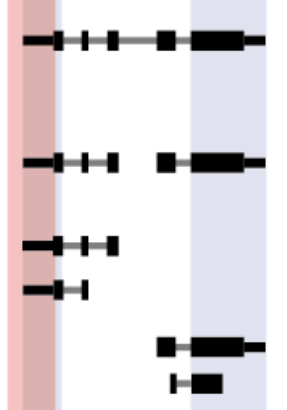

F

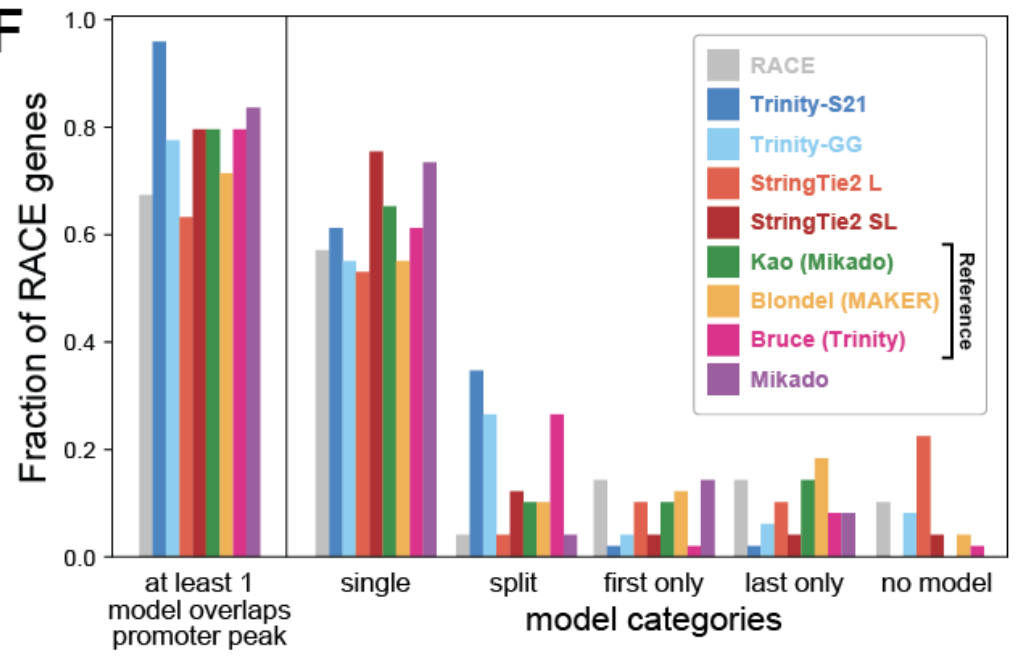

Omni-ATAC-Seq
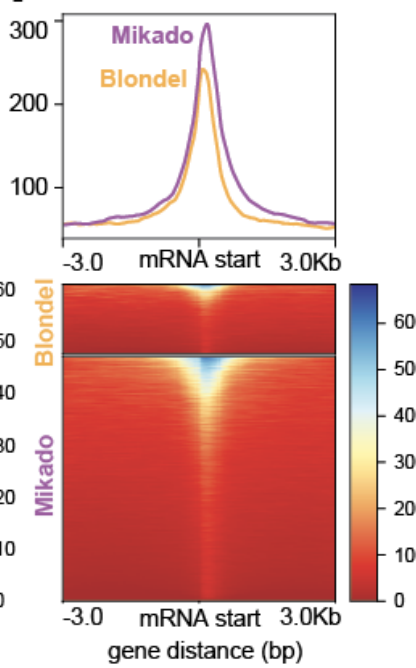

J

all

all

$\mathbf{S 1 4}$

S15

$\mathrm{S} 17$

$\mathrm{S} 18$

S19

S19

S20

S21

\$22

S23

$300 \quad 524$

S25

S26

S27

gene distance (bp)

ATAC peak type proportion by stage

\begin{tabular}{|c|ccc|}
\hline 0.182 & 0.512 & 0.201 & 0.094 \\
\hline 0.266 & 0.399 & 0.211 & 0.1 \\
\hline 0.291 & 0.386 & 0.201 & 0.098 \\
\hline 0.288 & 0.39 & 0.201 & 0.098 \\
\hline 0.247 & 0.424 & 0.21 & 0.098 \\
\hline 0.239 & 0.434 & 0.214 & 0.094 \\
\hline 0.221 & 0.452 & 0.216 & 0.092 \\
\hline 0.208 & 0.463 & 0.219 & 0.092 \\
\hline 0.187 & 0.487 & 0.223 & 0.087 \\
\hline 0.18 & 0.516 & 0.215 & 0.076 \\
\hline 0.236 & 0.473 & 0.198 & 0.077 \\
\hline 0.162 & 0.525 & 0.217 & 0.082 \\
\hline 0.158 & 0.528 & 0.216 & 0.084 \\
\hline 0.171 & 0.514 & 0.213 & 0.087 \\
\hline 0.168 & 0.52 & 0.213 & 0.085 \\
\hline 0.178 & 0.513 & 0.209 & 0.086 \\
\hline
\end{tabular}


bioRxiv preprint doi: https://doi.org/10.1101/2021.09.16.460328; this version posted September 18, 2021. The copyright holder for this preprint (which was not certified by peer review) is the author/funder, who has granted bioRxiv a license to display the preprint in perpetuity. It is made available under aCC-BY-NC-ND 4.0 International license.

Fig. 3: Time-course RNA-Seq of Parhyale embryos
A) RNA-Seq protocol overview. Triplicate libraries were generated for four developmental stages.
B) RNA-Seq transcriptome assembly and merging pipeline. Short read, long read, and long+short read transcriptomes were generated and then merged with additional transcripts from previous publications into a Mikado merged transcriptome.
C) Representative embryo images from clutches used for RNA-Seq.
D) BUSCO score of transcriptomes assembled for this study compared to previous reference data.
E) Overview of strategy for model evaluation using manual annotation of RACE genes.
F) Results of RACE gene model evaluation, plotted as a fraction of RACE genes that fit into several categories.
G) Comparison of number of gene models of different categories between Blondel reference annotation and Mikado merged transcriptome.
H) Plot comparing RNA-Seq signal pileups between Blondel mRNA starts and Mikado mRNA starts.
I) Plot comparing Omni-ATAC-Seq signal pileups between Blondel mRNA starts and Mikado mRNA starts.
J) Categorization of Omni-ATAC-Seq peaks in each stage-specific ATAC-Seq library by their position relative to Mikado gene models. Distal intergenic peaks are defined as those greater than $10 \mathrm{~kb}$ away from the nearest gene. 
bioRxiv preprint doi: https://doi.org/10.1101/2021.09.16.460328; this version posted September 18, 2021. The copyright holder for this preprint (which was not certified by peer review) is the author/funder, who has granted bioRxiv a license to display the preprint in perpetuity. It is made

A NucleoATAC
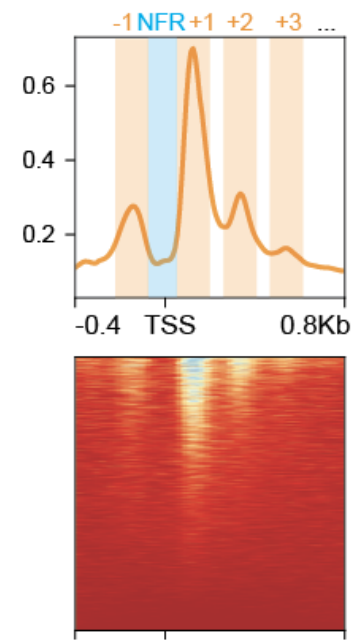

-0.4 TSS $\quad 0.8 \mathrm{~Kb}$ gene distance $(\mathrm{bp})$
B NucleoATAC

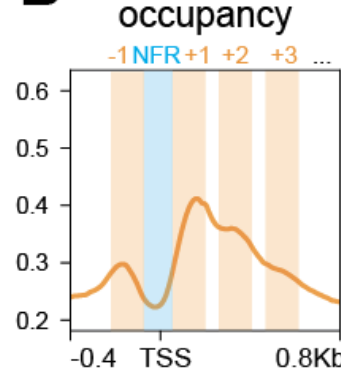

-1.4
-1.2
-1.0
-0.8
-0.6
-0.4
-0.2
0.0

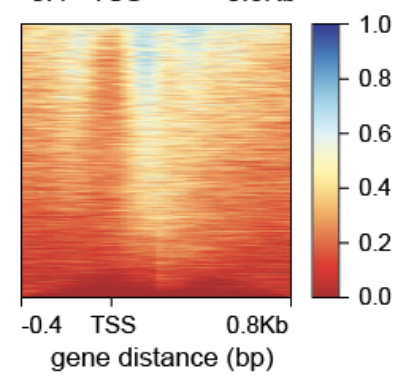

D JASPAR CORE (all species)

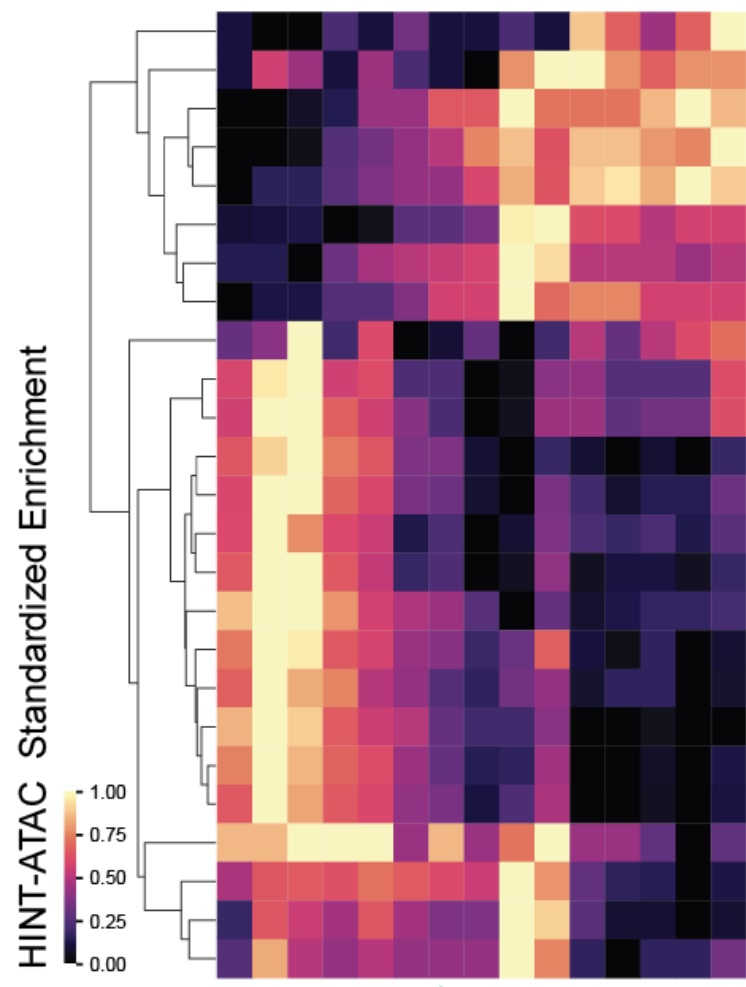

Dof3

eor-1

Tcf12

Ahr::Arnt

ARG80

MSN2/ MSN4/ RGM1

MET28

SKN7

HAL9

MNB1A/ PBF

blmp-1

Deaf1

OBP3

Dof2

YAP5

ct

HAP2

ZNF354C

Six4/ so

Optix

FZF1

lin-14

MOT3

AGL55

achi/ hth/ vis

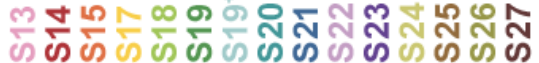

C

Genrich peaks

NucleoATAC NFRs

(nucleosome-free regions)

[0 - 334]

ATAC-Seq signal

NucleoATAC [0 - 104] insertions

NucleoATAC ${ }^{[0-3.69]}$

nucleosome occpancy

HINT-ATAC signal

HINT-ATAC

L footprints $[0-76]$

reads

Mikado

transcript models

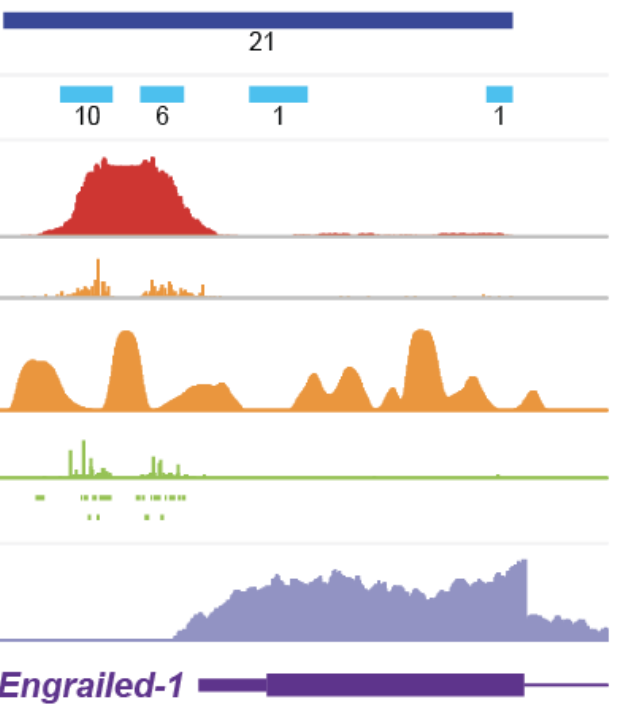

E JASPAR CORE (Drosophila)

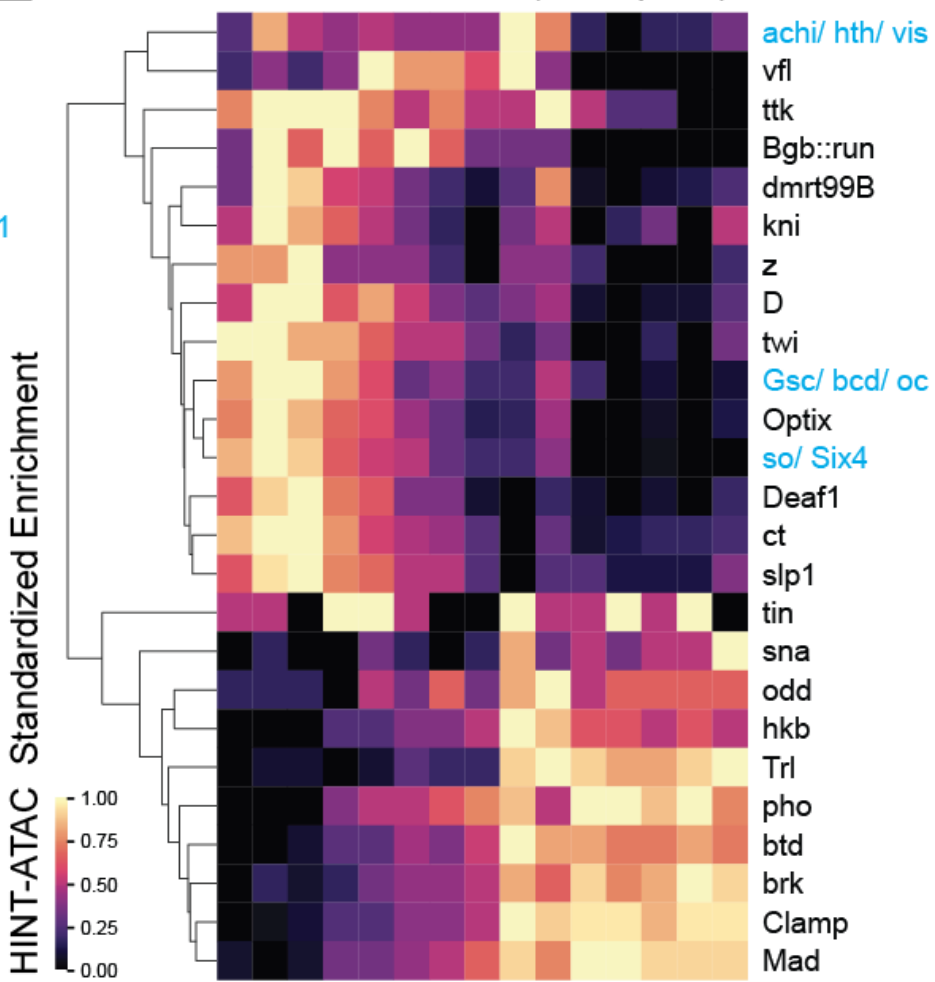

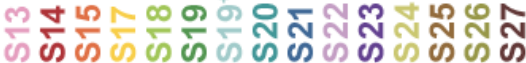




\section{Fig.4: Inference of nucleosome positioning and transcription factor binding from}

\section{Omni-ATAC-Seq data}

A) NucleoATAC smoothed signal at mRNA starts genome-wide

B) Inferred nucleosome occupancy at mRNA starts genome-wide. Inferred nucleosome annotations on A and B added based on observed signal.

C) Visualization of NucleoATAC, HINT-ATAC, Omni-ATAC, and RNA-Seq data at the inferred Engrailed-1 promoter.

D) Summary of standardized enrichment of JASPAR CORE position-weight matrices (PWMs) among HINT-ATAC identified footprints across developmental stages. The top 25 most-enriched PWMs are displayed. Color is based on standardized enrichment, where minimum and maximum enrichment within each row are set to 0 and 1 , respectively. PWMs marked in blue had identical enrichment ratios within each group at all timepoints, likely due to having highly similar PWMs.

E) Summary of top 25 most-enriched Drosophila PWMs from the JASPAR CORE database. 
bioRxiv preprint doi: https://doi.org/10.1101/2021.09.16.460328; this version posted September 18, 2021. The copyright holder for this preprint

(which was not certified by peer review) is the author/funder, who has granted bioRxiv a license to display the preprint in perpetuity. It is made

A t-SNE by IDE2 model fit max

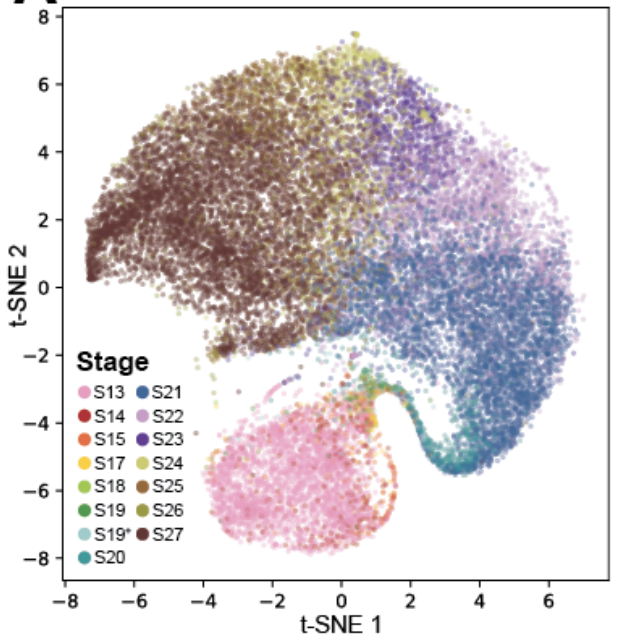

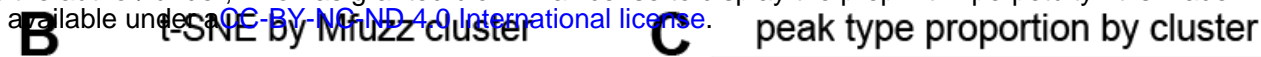

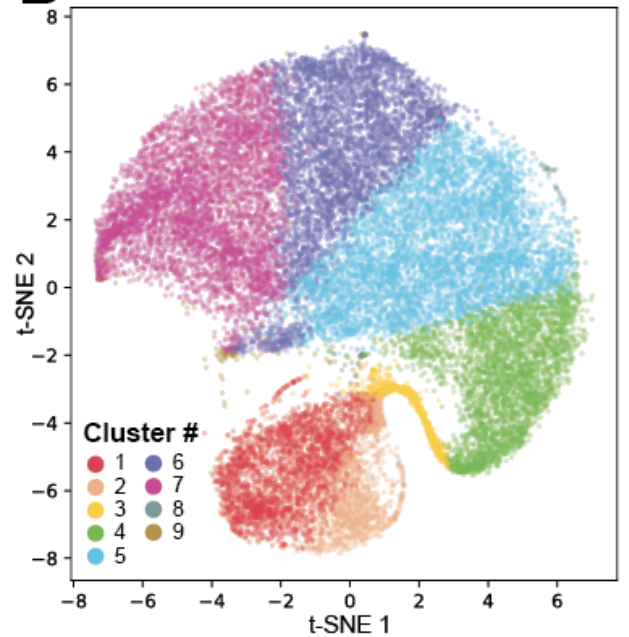

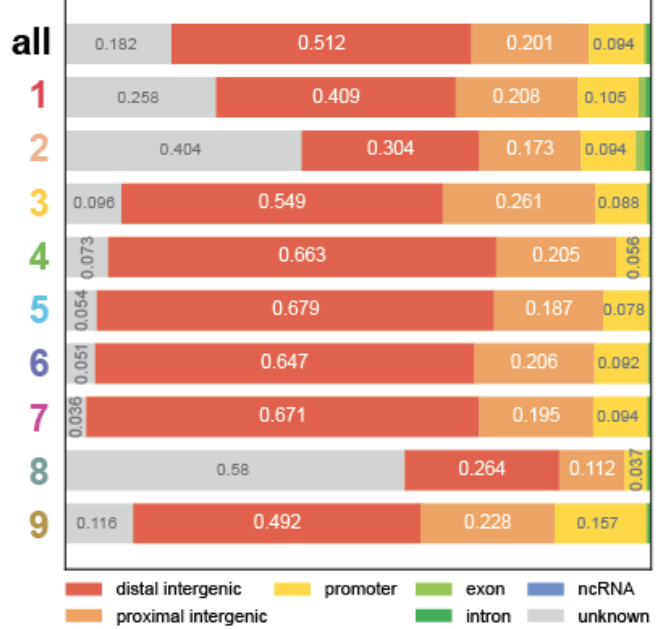

Deak accessibility
(top 1000 peaks)
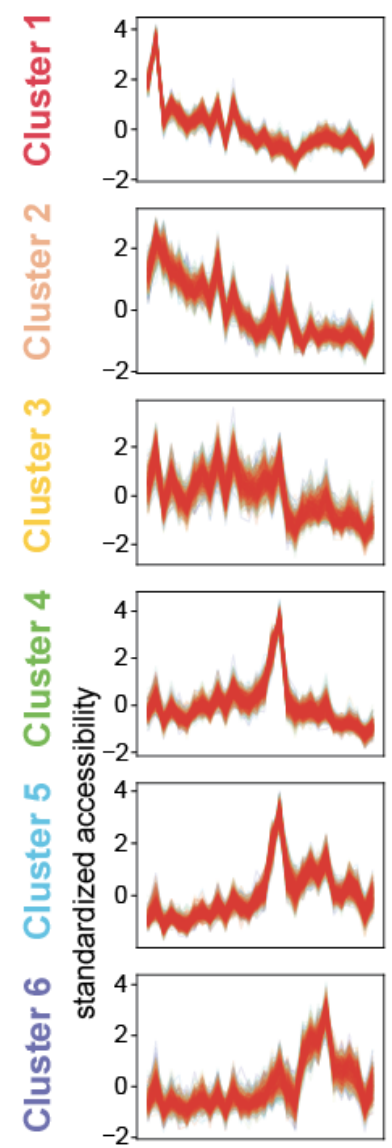

는

$$
\text { 高 }
$$$$
\text { 苟 }
$$

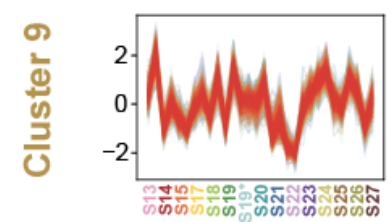

developmental stage
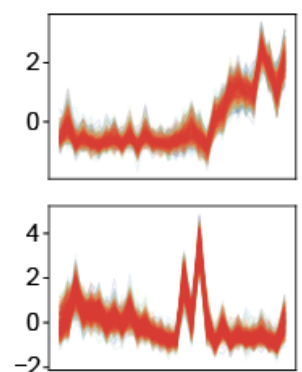

$\mathbf{E}_{\text {(top } 200 \text { peaks) }}^{\text {IDE } 200 \text { fit }}$

$\mathbf{F}$ IDE2 model $\mathbf{G}$
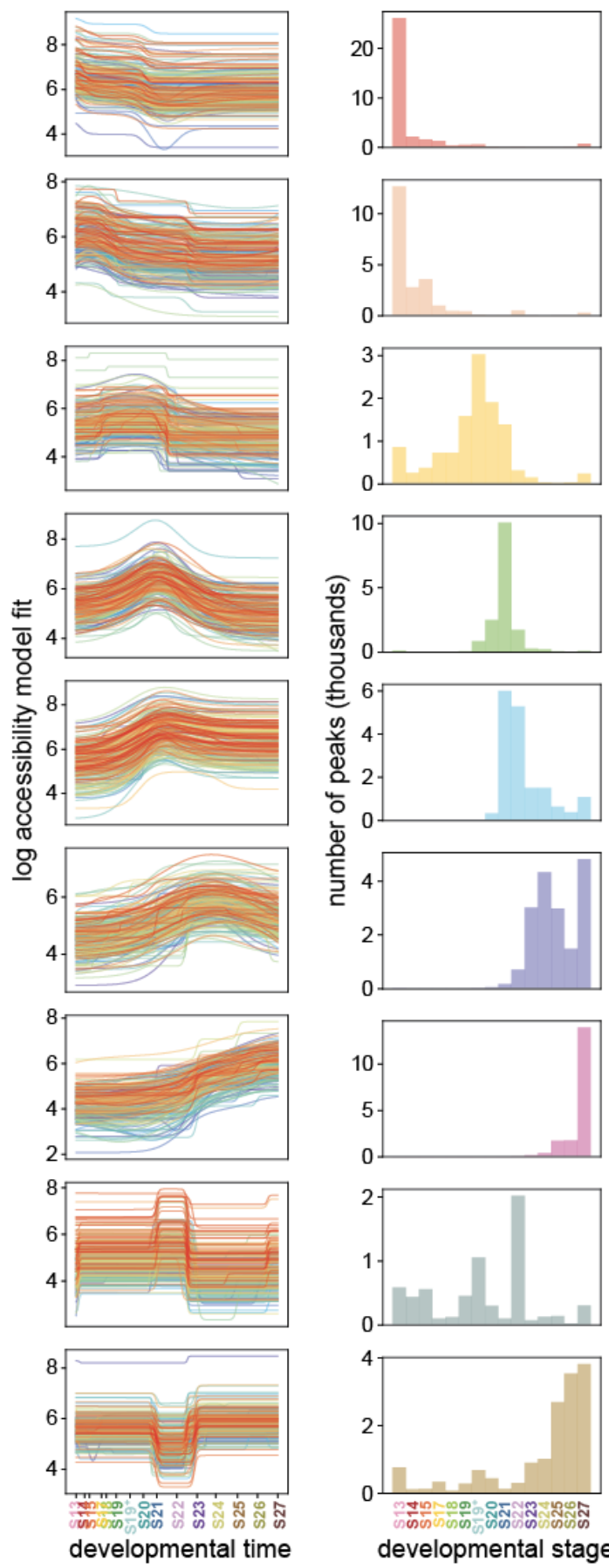
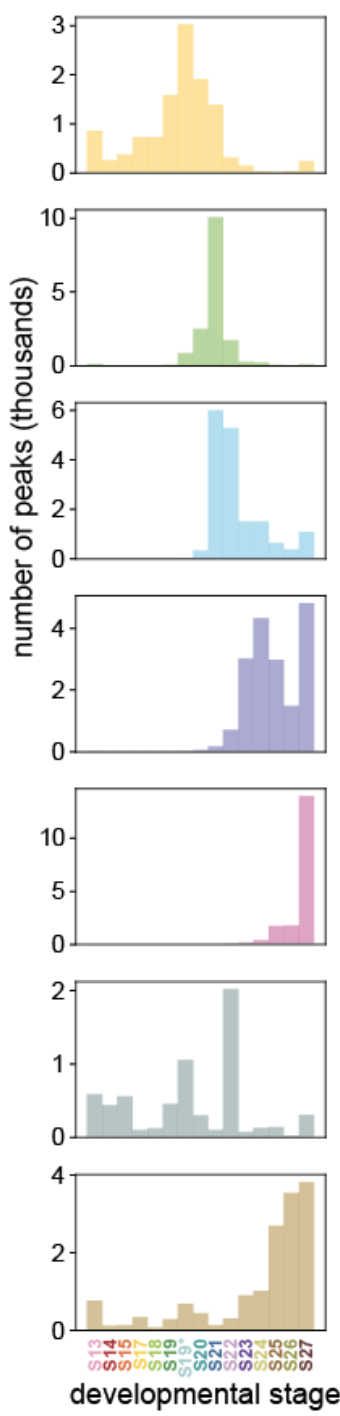

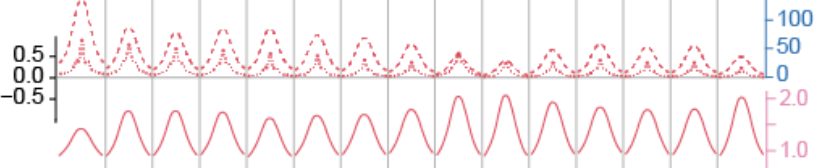

0.5
0.0
-0.5

$0.5-$
0.0.
-0.5

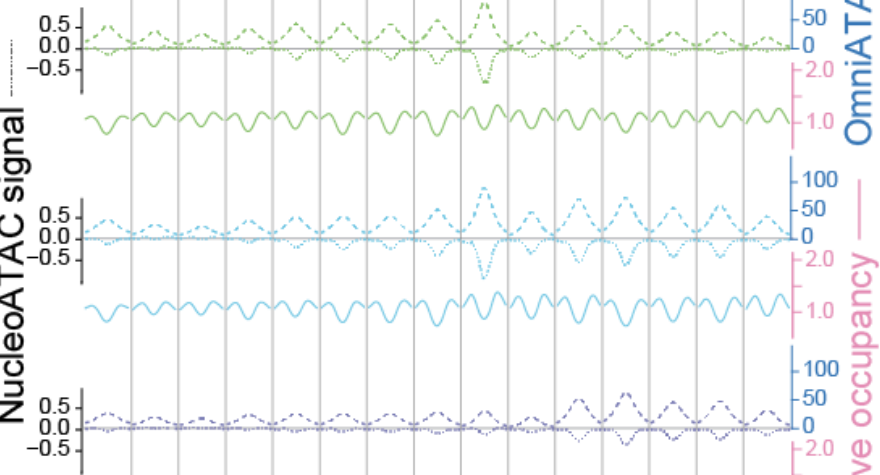

$0.5-$

-0.5 -

0.5.
0.0.
-0.5

$100 \stackrel{2}{\mathrm{Z}}$

0.5.

-0.5 -

$-0.4 \mathrm{~kb}-++0.4 \mathrm{~kb}$

peak center

genomic coordinate per box 


\section{Fig. 5: Identification and classification of regulatory element clusters}

A) t-SNE plot of the top 10,000 most statistically significant peaks identified by ImpulseDE2, colored by IDE2 max fit.

B) t-SNE plot of the same points from A colored by Mfuzz cluster.

C) Distribution of peak position categories by Mfuzz cluster.

D) Standardized accessibility plot for top 1000 peaks with strongest membership in each of the 9 clusters. Each library is plotted as a separate point along the line plot.

E) ImpulseDE2 model fits for the top 200 peaks with strongest membership in each of the 9 clusters.

F) Histogram of when all peaks in each cluster achieve their maximum accessibility as calculated by IDE2 max fit.

G) Line plots showing Omni-ATAC accessibility (dashed line, black axis), NucleoATAC signal (dotted line, blue axis), and relative NucleoATAC histone occupancy (solid line, pink axis) for peaks in each cluster across time. Each box contains a line plot summarizing each of the three signals at all peaks within that cluster with respect to a given developmental Omni-ATAC-Seq library. The center of each line plot reflects the average signal at the center of all Omni-ATAC-Seq peaks in that cluster at that developmental stage. Signal is visualized at $0.4 \mathrm{~kb}$ upstream and downstream of peak centers. Within each signal type, the axes across line plots for each cluster are identical. 
bioRxiv preprint doi: https://doi.org/10.1101/2021.09.16.460328; this version posted September 18, 2021. The copyright holder for this preprint

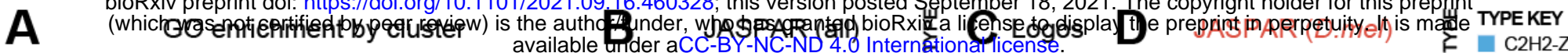

$\frac{5}{\frac{2}{0}}$

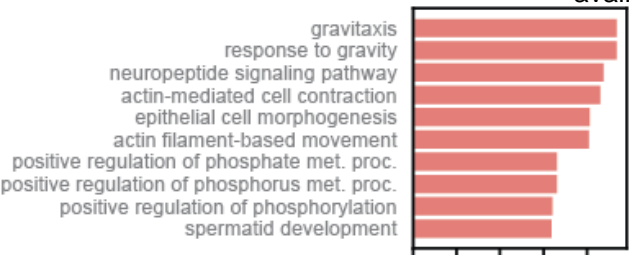

0.00 .51 .01 .52 .0

cardioblast cell fate commitment regulation of neuron projection arborization response to auditory stimulus
sperm mitochondrion organization peroxisom convergent extension peroxisomal transport regulation of nervous system process regulation of establishment of planar polarity

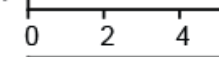

posterior head segmentation neuroblast fate determination neuroblast fate commitment head segmentation regulation of establishment of planar polarity
regulation of animal organ morphogenesis neuroblast differentiation muscle cell fate commitiment sensory organ boundary specification

oenocyte differentiation segmental identity, head anterior head segmentation anterior head segmentation head segmentation posterior head segmentation posterior head segmentation determination of imaginal disc primordium regulation of cell fate specification neuroblast development

cardiac muscle tissue development
specification of segmental identity, head specification of segmental identity, head
muscle cell fate commitment regulation of establishment of planar polarity head segmentation antennal development neuroblast development germ-band shortening cardiocyte differentiation
formation of anatomical boundary
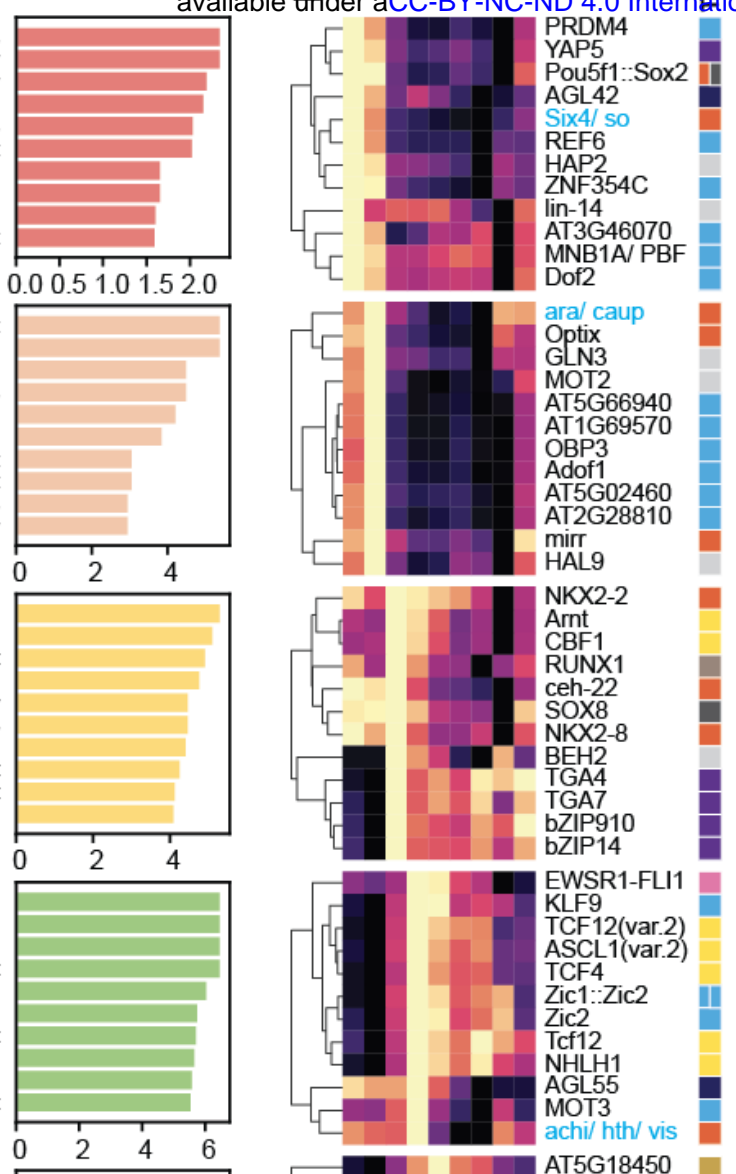

EWSR1-FLI1

KLF9

ASCL1(var.2)

TCF4 4 (var.2)

Zic1::Zic2

Zic2

Tcf12

NHLH1

AGL55

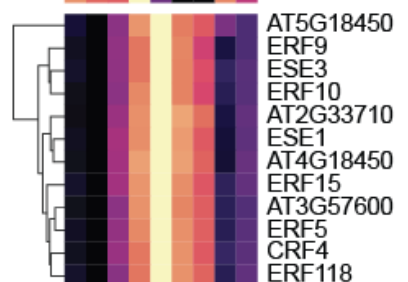

AT5G1845

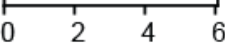

$\omega$

maint. of epithelial integrity, open tracheal sys. regulation of establishment of planar polarity antennal development formation of anatomical boundary formation of animal organ boundary regulation of animal organ morphogenesis midgut development

epithelial structure maintenance

actin fil animal organ formation

actin filament-based movement

neuropeptide signaling pathway cell-cell adhes. via plasma membrane synaptic target recognition
es. via plasma membrane... N

(1)

$\frac{0}{0}$ cell-cell adhesion
cell adhesion
biological adhesion
mating behavior
GPCR signaling pathway neuron recognition

perant conditioning

$\frac{\infty}{\frac{1}{3}}$ $\begin{array}{lllll}0 & 1 & 2 & 3 & 4\end{array}$
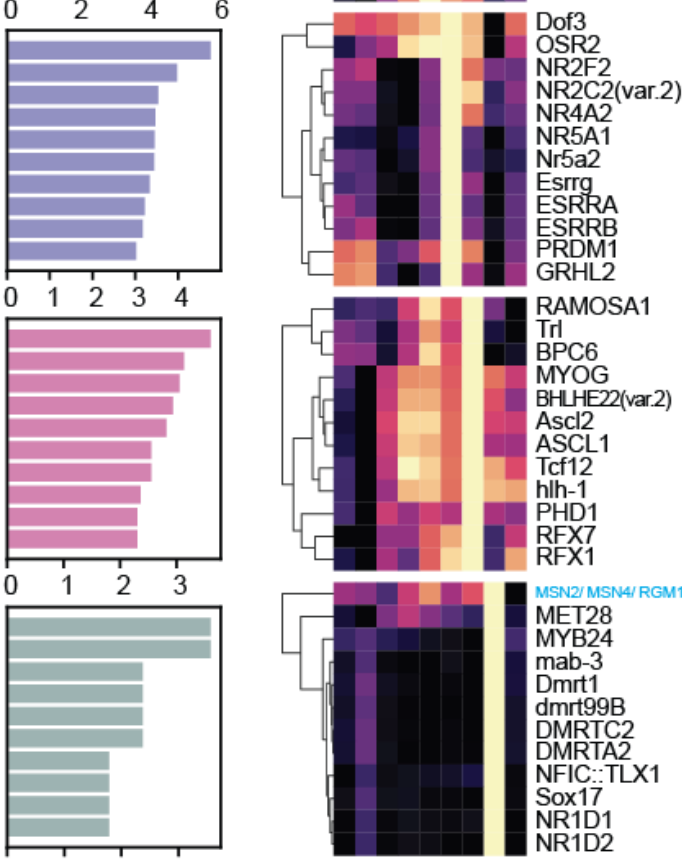

XISTH

MET28

MYB24

mab-3

Dmrt1

DMRTC2

DMRTA2

Sox17

NR1D1
NR1D2

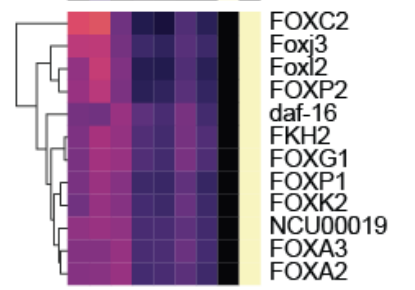

ปกำ
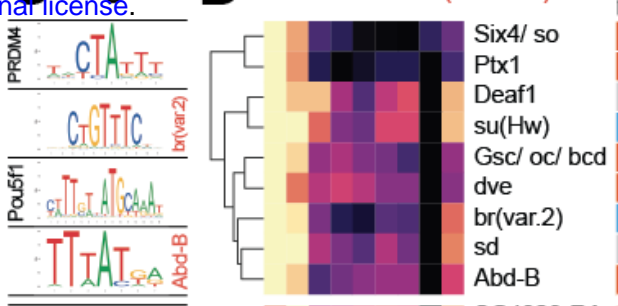

E $\mathrm{C} 2 \mathrm{H} 2-\mathrm{ZF}$

NR C4-ZF

DM IZF

FKH

Homeo

bZIP

bHLH

ERF

HMG

Try-C

- SMAD

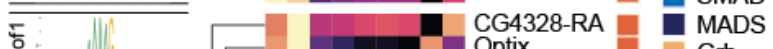

ICATATA
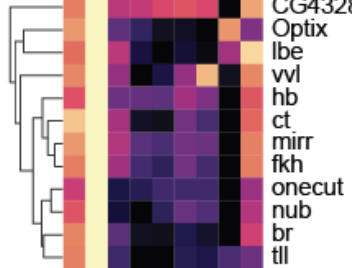

Grh

Runt

Other

HINT-ATAC

Standardized

Enrichment

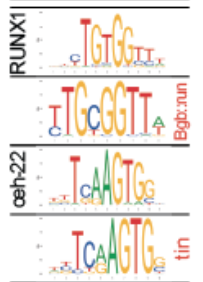

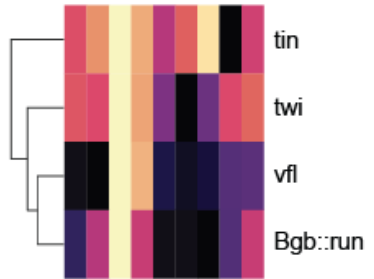

$-1.0$

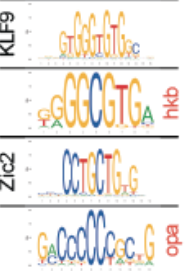

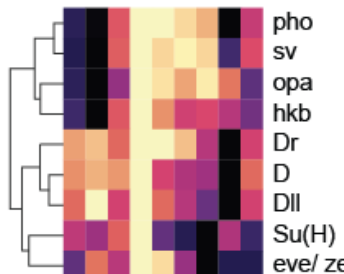

要

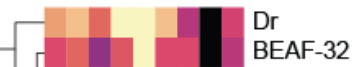

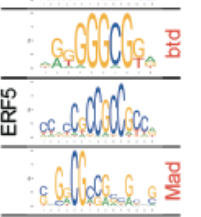
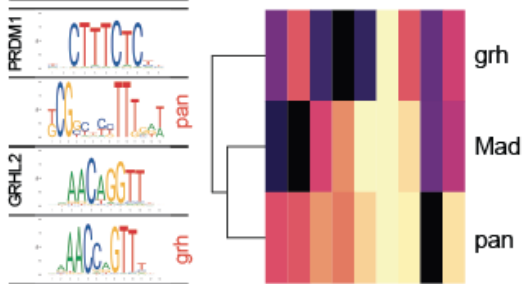

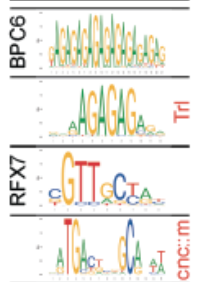
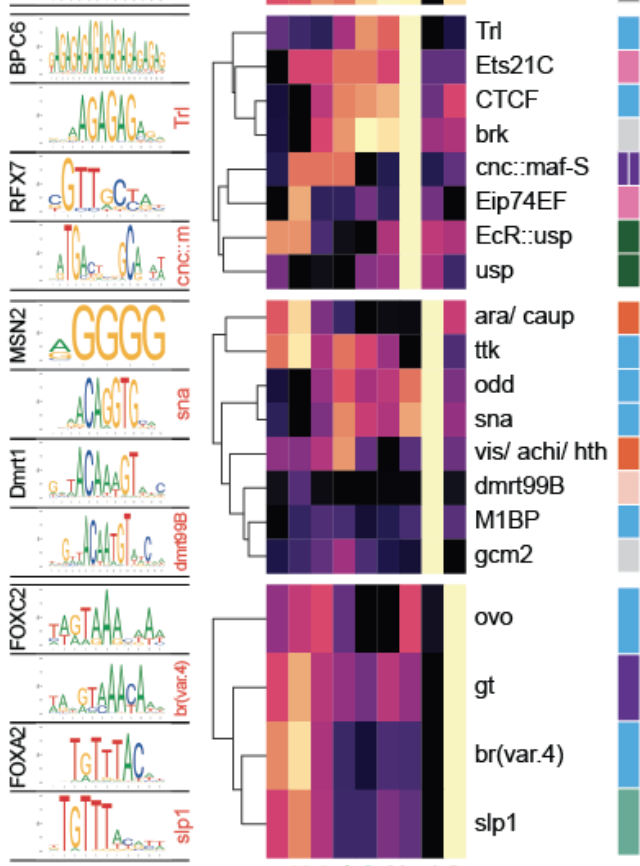

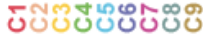


Fig. 6: Functional annotation of clusters using GO enrichment and transcription factor binding prediction

A) GO enrichment per cluster based on the nearest gene for each peak. For each peak in each cluster, we identified the nearest gene and extracted the Drosophila OrthoFinder gene name for that gene. We performed GO enrichment using Drosophila GO terms, using the list of all OrthoFinder gene names found in our genome as the background for the analysis. Bar charts display fold enrichment for each $\mathrm{GO}$ term. Gray text represents $\mathrm{GO}$ terms that did not have a significant $p$-value $(p<0.05)$ after false discovery rate $(F D R)$ correction.

B) HINT-ATAC enrichment per cluster for JASPAR CORE PWMs. For each cluster, we identified all unique TF footprints across all 15 timepoints found within peaks of that cluster and performed enrichment relative to a randomly-selected background. We show the top 12 PWMs with the greatest enrichment for each cluster that also had the highest enrichment in that cluster relative to other clusters. Colored boxes represent the transcription factor family of each transcription factor in the clustermap.

C) JASPAR motif logos for select pairs of transcription factors between non-Drosophila and Drosophila TFs with similar motifs as determined by STAMP alignment. For each cluster, two pairs of transcription factors are shown.

D) HINT-ATAC enrichment per cluster for Drosophila JASPAR CORE PWMs. Some clusters had fewer than 12 PWMs for which that cluster had the highest enrichment. 
bioRxiv preprint doi: https://doi.org/10.1101/2021.09.16.460328; this version posted September 18, 2021. The copyright holder for this preprint (which was not certified by peer review) is the author/funder, who has granted bioRxiv a license to display the preprint in perpetuity. It is made available under aCC-BY-NC-ND 4.0 International license.
A
S19 vs S13 S21 vs S19
S23 vs $\mathrm{S21}$
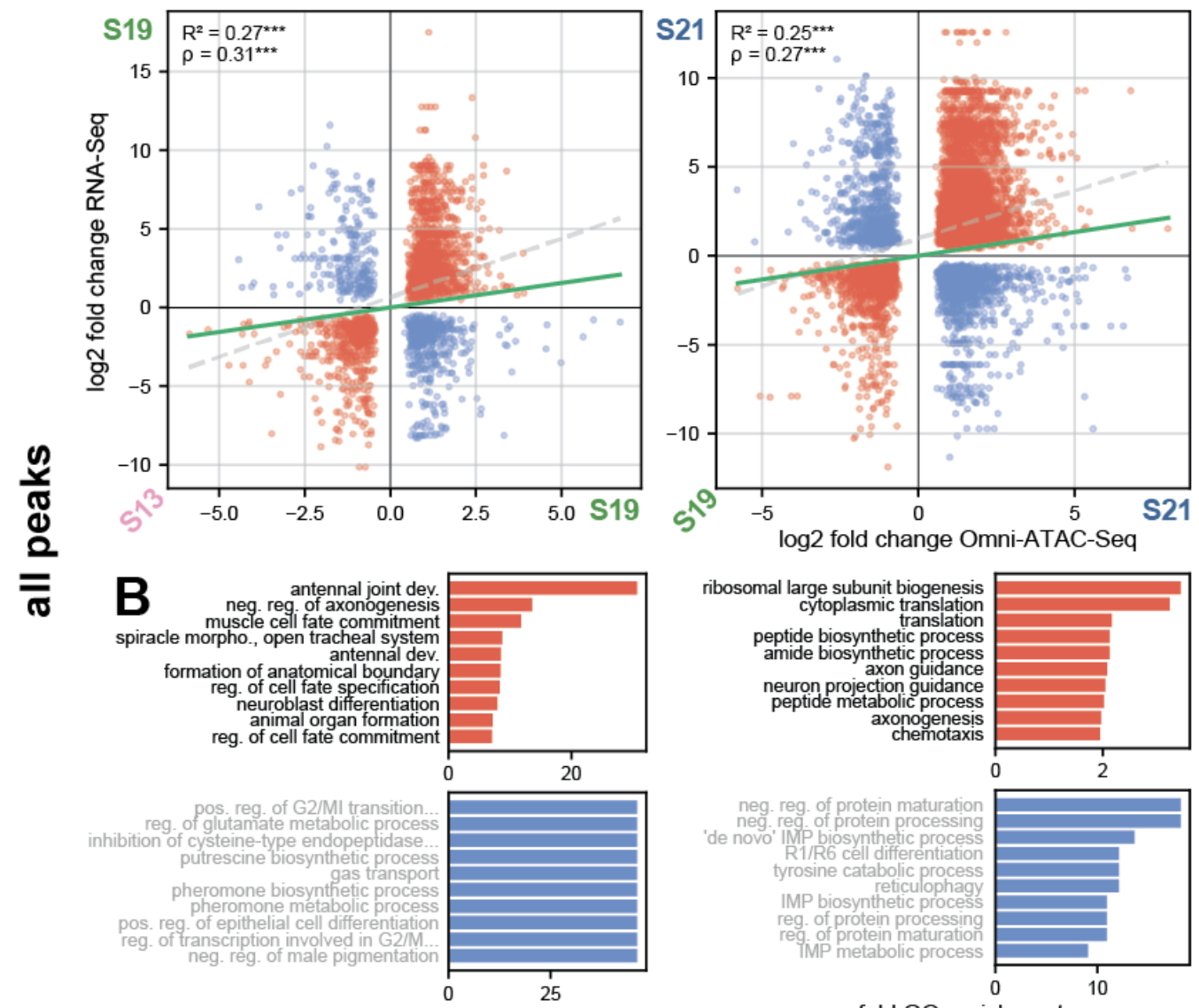

fold GO enrichment
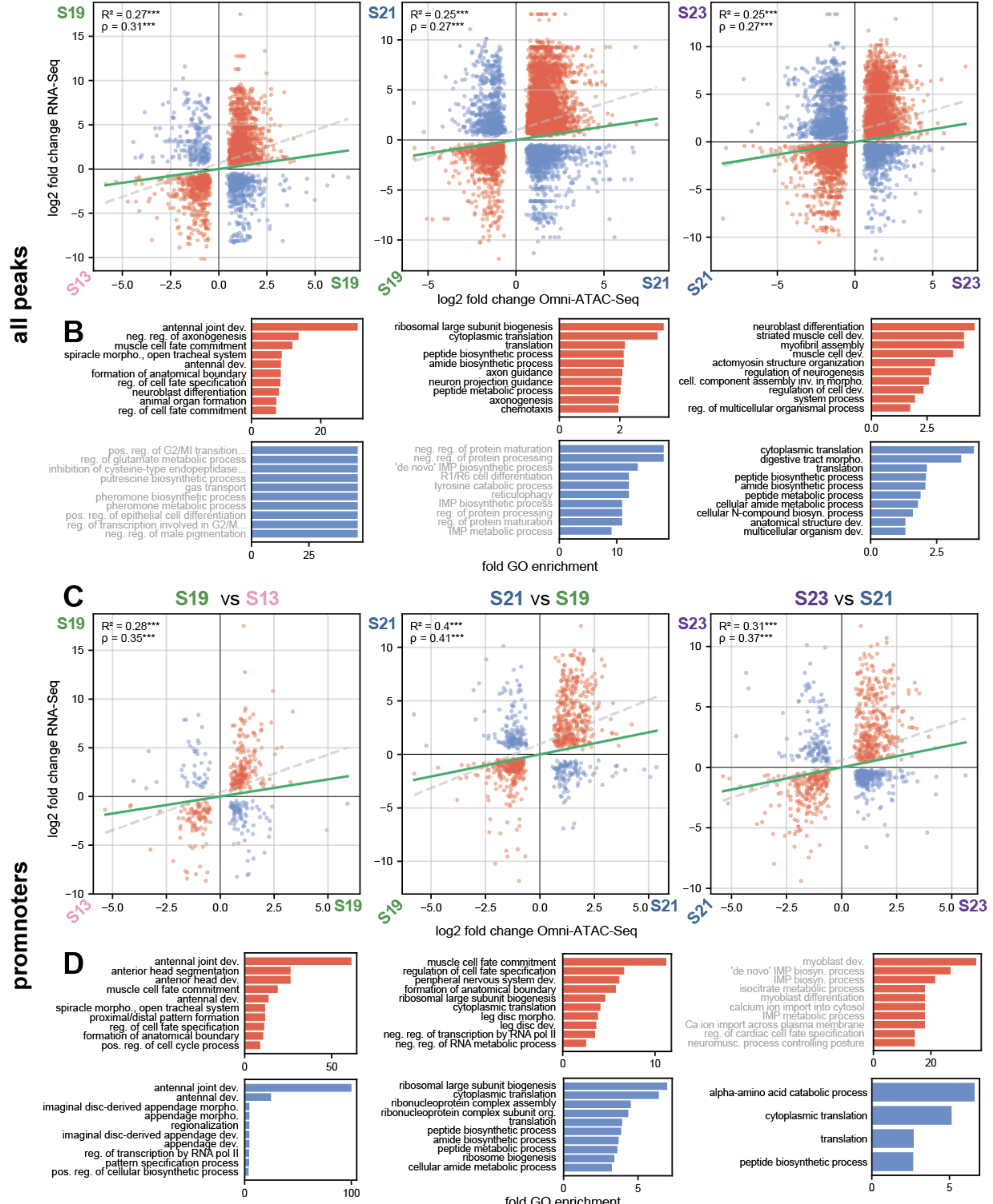

alpha-amino acid catabolic process

cytoplasmic translation translation

peptide biosynthetic process fold GO enrichment

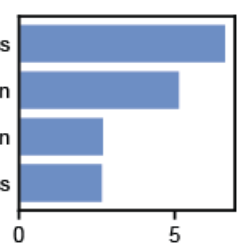


bioRxiv preprint doi: https://doi.org/10.1101/2021.09.16.460328; this version posted September 18, 2021. The copyright holder for this preprint (which was not certified by peer review) is the author/funder, who has granted bioRxiv a license to display the preprint in perpetuity. It is made available under aCC-BY-NC-ND 4.0 International license.

Fig. 7: Correlation between RNA-Seq and Omni-ATAC-Seq

A) Correlation between log fold change RNA-Seq and log fold change Omni-ATAC-Seq at all peaks in sequential pairwise comparisons of developmental stages. Positive axis reflects higher expression at the later developmental stage for each plot. Peaks colored in red reflect concordant relationships between log fold change in RNA-Seq and Omni-ATAC-Seq; blue peaks reflect discordant relationships.

B) GO-term enrichment for gene-peak pairs with concordant or discordant expression and accessibility log2 fold change, for all peaks. Gene lists were extracted based on the nearest Mikado gene to each peak.

C) Correlation between log fold change RNA-Seq and log fold change Omni-ATAC-Seq at all peaks in each developmental stage. Positive axis reflects higher expression at the later developmental stage for each plot.

D) GO-term enrichment for gene-peak pairs with concordant or discordant expression and accessibility log2 fold change, for promoter peaks.

$\left({ }^{* * *}=p<0.001\right)$ 
bioRxiv preprint doi: https://doi.org/10.1101/2021.09.16.460328; this version posted September 18, 2021. The copyright holder for this preprint (which was not certified by peer review) is the author/funder, who has granted bioRxiv a license to display the preprint in perpetuity. It is made available under aCC-BY-NC-ND 4.0 International license.

A

\section{Parhyale} hawaiensis

C

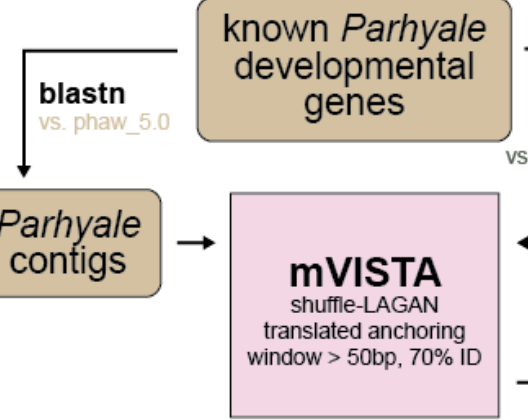

E Minos reporter assay

$=$
$\vdots$
$\vdots$
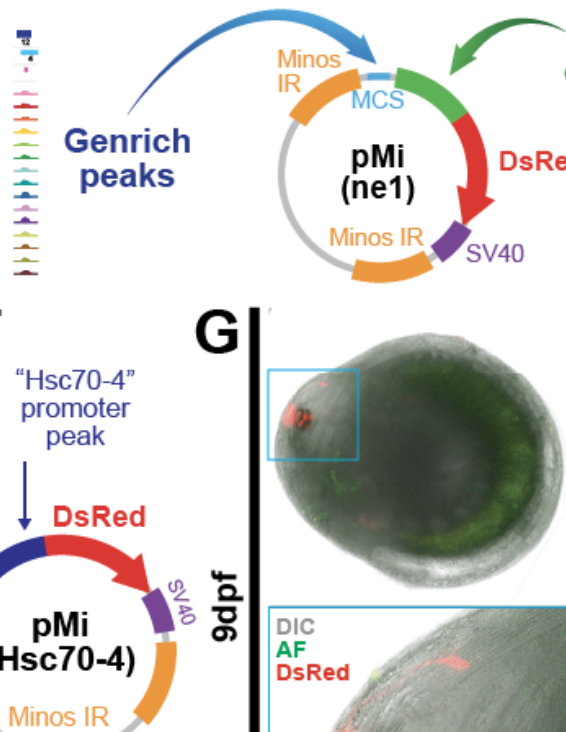

\section{$\mathbf{F}$ (Hsc70-4) \\ "Hsc70-4" promoter

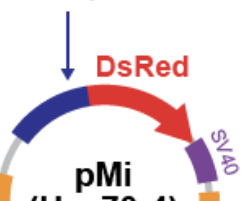

Predicted endogenous promoter

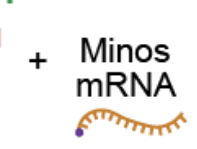

D

Genrich peaks NucATAC NFR
Hyalella azteca

\section{$\rightarrow$ VISTA cons.}

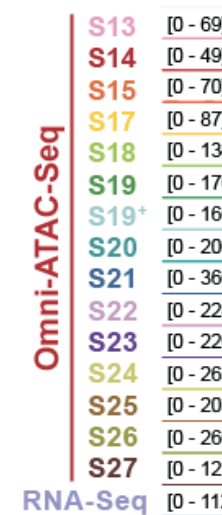

\section{Sequence} conservation
RNA-Seq [0-112]

Mikado gene models

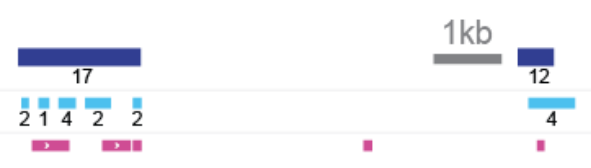

mikado.phaw_50.282861eG239.1
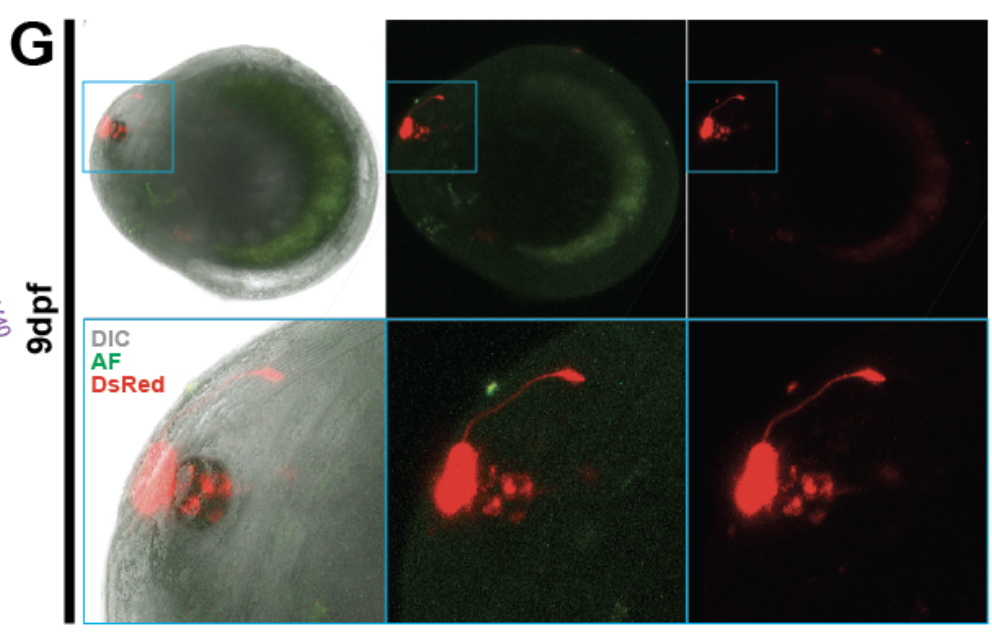

PhHsp70 OR minimal promoter

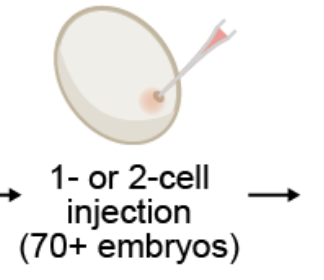

DsRed

\section{fluorescent embryos/ expression patterns \\ Screen for $1 \mathrm{x} /$ day on 3-10dpf} $\mathrm{H}$

Genrich peaks NucATAC NFR VISTA cons.
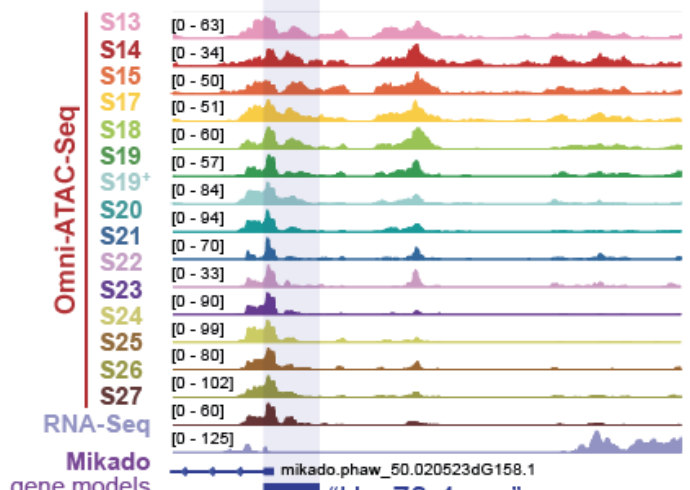
gene models

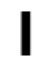

"Hsp70-p2" Genrich peak
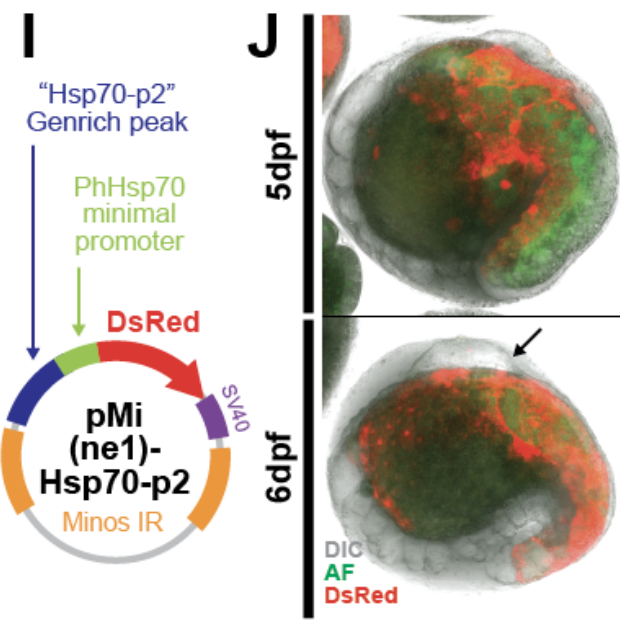

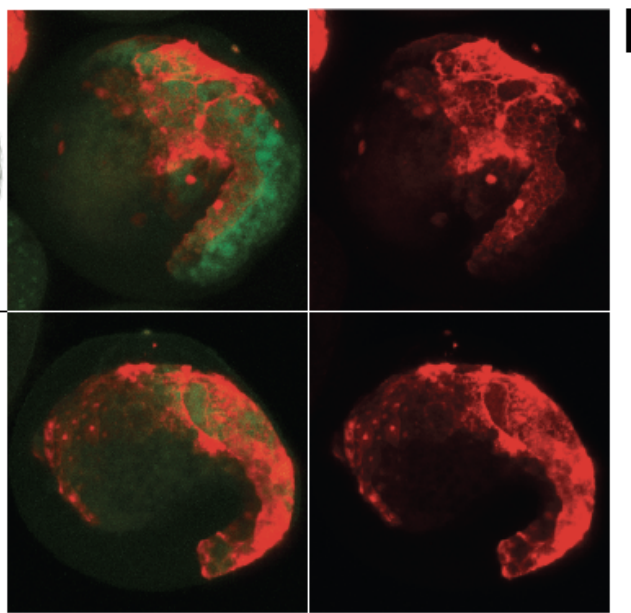

$K_{\text {G }}$

Genrich peaks NucATAC NFR VISTA cons.

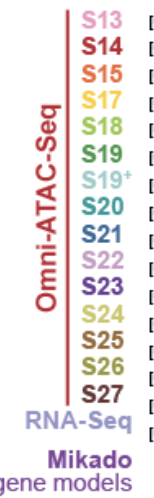

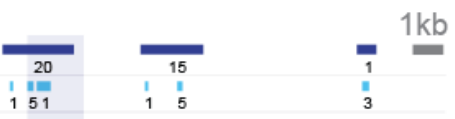
"Hsc70-4 pro"
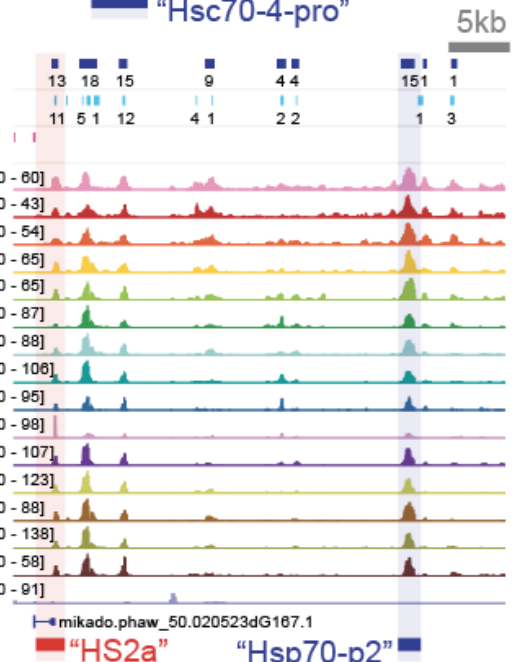


\section{Fig. 8: Minos transposase reporter assay identification of novel regulatory elements}
A) Adult female Parhyale hawaiensis.
B) Adult female Hyalella azteca.
C) Pipeline for identification of orthologous genomic regions between Hyalella and Parhyale for VISTA sequence conservation analysis.
D) Visualization of Genrich peaks, NucleoATAC nucleosome-free regions (NFRs), VISTA conservation, Omni-ATAC-Seq signal, RNA-Seq signal, and Mikado gene models at the putative Sp69 promoter peak.
E) Minos transposase reporter assay and screening approach.
F) Plasmid schematic for the pMi(ne1)-Hsp70-p2 reporter.
G) Expression of pMi(ne1)-Hsp70-p2 reporter at 5 and 6dpf showing strong expression in the yolk.
$\mathrm{H})$ The genomic region of the Hsp70-p2 peak relative to the HS2a element. The PhHsp70 minimal promoter used in this construct is located within the HS2a element.
I) Plasmid schematic for the pMi(Hsc70-4) reporter.
J) Expression of the pMi(Hsc70-4) reporter in neurons associated with the eye of a 9hpf embryo. Bottom row shows zoomed-in image of the head region. A clear projection of the neurons can be seen.
K) The genomic region of the Hsc70-4 promoter peak. 
bioRxiv preprint doi: https://doi.org/10.1101/2021.09.16.460328; this version posted September 18, 2021. The copyright holder for this preprint (which was not certified by peer review) is the author/funder, who has granted bioRxiv a license to display the preprint in perpetuity. It is made available under aCC-BY-NC-ND 4.0 International license.

Table 1: Plasmids tested using Minos transgenesis

\begin{tabular}{|c|c|c|c|c|c|c|}
\hline Plasmid & Туре & $\begin{array}{l}\text { Gene - Drosophila } \\
\text { expression }\end{array}$ & \# Inj. & \# Surv. & \# DsRed+ & Phenotype \\
\hline pMi(gm) & promoter & $\begin{array}{l}\text { N/A - Parhyale muscle } \\
\text { reporter PhMS }\end{array}$ & 109 & 39 & 24 & $\begin{array}{l}\text { Green muscle } \\
\text { expression }\end{array}$ \\
\hline pMi(retn) & promoter & $\begin{array}{l}\text { retained-expressed in } \\
\text { some neurons }\end{array}$ & 240 & 104 & 5 & $\begin{array}{l}\text { No consistent } \\
\text { phenotype }\end{array}$ \\
\hline pMi(SVP) & promoter & $\begin{array}{l}\text { seven up - expressed in } \\
\text { ventral neuroblasts }\end{array}$ & 122 & 31 & 0 & None \\
\hline pMi(su-s-2) & pro + peak & $\begin{array}{l}\text { suppressor of sable - } \\
\text { embryonic ubiquitous } \\
\text { expression }\end{array}$ & 127 & 57 & 7 & $\begin{array}{l}\text { No consistent } \\
\text { phenotype }\end{array}$ \\
\hline $\mathrm{pMi}(\mathrm{Rx})$ & pro + peak & $\begin{array}{l}\text { retinal homeobox - } \\
\text { expressed in various neural } \\
\text { tissues }\end{array}$ & 74 & 28 & 0 & None \\
\hline pMi(Hsc70-4) & promoter & $\begin{array}{l}\text { Heat shock } 70 \text { complex } \\
\text { protein } 4 \text { - embryonic } \\
\text { ubiquitous }\end{array}$ & 212 & 99 & 22 & $\begin{array}{l}\text { Neurons } \\
\text { associated with } \\
\text { the eye }\end{array}$ \\
\hline $\mathrm{pMi}(\mathrm{En} 1)$ & promoter & $\begin{array}{l}\text { engrailed - parasegment } \\
\text { stripes }\end{array}$ & 71 & 33 & 0 & None \\
\hline pMi(En1)-p1 & pro + peak & $"$ & 67 & 46 & 0 & None \\
\hline pMi(En1)-p1.5 & pro + peak & $"$ & 70 & 51 & 0 & None \\
\hline pMi(En1)-p5 & pro + peak & $"$ & 68 & 58 & 1 & $\begin{array}{l}\text { Faint expression } \\
\text { domain }\end{array}$ \\
\hline $\mathrm{pMi}(\mathrm{Sp69})$ & promoter & Sp69 - leg gap gene & 74 & 56 & 0 & None \\
\hline pMi(Sp69)-p1 & pro + peak & $"$ & 50 & 30 & 0 & None \\
\hline pMi(Sp69)-p1.5 & pro + peak & $"$ & 86 & 32 & 3 & $\begin{array}{l}\text { No consistent } \\
\text { phenotype }\end{array}$ \\
\hline pMi(Sp69)-p2.5 & pro + peak & $"$ & 71 & 35 & 0 & None \\
\hline pMi(Sp69)-p3 & pro + peak & $"$ & 241 & 121 & 8 & Head, leg, gill \\
\hline pMi(ne1)-Hsp70-p1 & pro + peak & Hsp70 complex & 45 & 26 & 0 & None \\
\hline pMi(ne1)-Hsp70-p2 & pro + peak & Hsp70 complex & 310 & 81 & 61 & Yolk cells \\
\hline
\end{tabular}




\section{Methods}

\section{Crustacean Cultures}

Parhyale hawaiensis were raised at $25^{\circ} \mathrm{C}$ and fed a diet of carrots, shrimp pellets, and Spirulina flakes in Tropic Marin artificial seawater with a salinity between 31-35ppm in plastic tanks.

\section{Embryo Cultures}

Embryos were collected using previously described protocols (Rehm et al., 2009), staged using the Browne et al. 2003 staging guide, and raised at $27^{\circ} \mathrm{C}$ in a humidity-controlled incubator in filter-sterilized ASW (FASW). For ATAC-Seq and RNA-Seq experiments, clutches of 15 or more embryos were collected and staged between S1-S6, as these timepoints are among the shortest and most morphologically identifiable during early development.

\section{Embryo Staging}

Two groups of five embryos each from a single clutch were used in the ATAC-Seq experiments, and three groups of five embryos each from a single clutch were used in the RNA-Seq experiments. Embryos of the correct stage were selected based on morphological characteristics as described in the Browne et al. staging guide, and any embryos with abnormal or asynchronous morphology were discarded. Morphologically representative embryos from the same clutch as used for the ATAC-Seq were photographed on ventral and lateral positions within 10 minutes of beginning the tagmentation procedure. Any remaining embryos were boiled briefly and fixed using the protocol described in (Rehm et al., 2009) and DAPI stained to further confirm staging. The process of staging embryos, imaging embryos, boiling, and mashing embryos for Omni-ATAC tagmentation were all performed within a 30 minute time interval for each developmental stage.

\section{RNA Sequencing}

Embryos for RNA isolation were homogenized in TriZol using a DWK Life Sciences (Kimble) Biomasher II Closed System Disposable Tissue Homogenizer, and RNA was isolated using the Zymo Direct-Zol Miniprep Plus kit. RNA quality was assessed based on fragment analysis using a Bioanalyzer. cDNA was generated from RNA using the TaKaRa SMART-Seq v4 Ultra Low-Input kit. cDNA was then sequenced using the Illumina NovaSeq to generate short reads. Using the Nanopore Direct cDNA barcoding kit (SQK-LSK109), cDNA was also sequenced on a Nanopore MinION flow cell to generate long reads.

\section{Transcriptome Assemblies}

Short-read RNA-Seq data was used to generate both de novo and genome-guided transcriptomes using Trinity. For genome-guided assembly, reads were mapped using HISAT2. Long-read RNA-Seq data was mapped to the most recent Parhyale genome (phaw_5.0) using minimap2 and assembled using StringTie2. A combined transcriptome using both HISAT2-mapped short reads and minimap2-mapped long reads was also generated using StringTie2. See Supplementary Methods for additional information about assembly parameters.

\section{Mikado Combined Transcriptome}

Short-read Trinity transcriptomes and two previously generated transcriptomes from Heather Bruce and from the Kao et al. manuscript were mapped to the phaw_5.0 genome using GMAP. Short-read transcriptomes and long-read StringTie2 transcriptomes were merged along with a previous genome annotation generated by Leo Blondel using the Mikado software. See supplementary methods for additional information about Mikado parameters. 


\section{Functional Annotation of Transcripts}

The merged Mikado combined transcriptome was annotated using eggNOG and an Orthofinder comparison to Drosophila. See Supplementary Methods for additional information about functional annotation.

\section{ATAC-Seq Data}

We performed conventional ATAC-Seq as per Buenrostro et al. 2013 for stages S13-S22. Data were used for benchmarking in comparison to Omni-ATAC data, but were not used for downstream analyses.

\section{Omni-ATAC-Seq Tagmentation}

Tagmentation was performed using the reagents described in the Corces et al. 2017 Omni-ATAC-Seq paper, with the following modifications. Instead of the Illumina Nextera TD buffer, 2x Tagmentation Buffer from Wang et al. 2013 was used (Corces et al., 2017; Wang et al., 2013). Homemade Tn5 enzymes were purified and received as a gift from Jase Gehring and assembled into Tn5 transposomes as per Picelli et al 2014, using adapters purchased from IDT with 5' phosphorylation (Picelli et al., 2014). Prior to adding $R S B+D$, embryos were washed $3 x$ using $1 X$ PBS. Embryos were mashed into a near-uniform solution using the tip of a low-retention p10 pipette, and the pipette was visually inspected for any remaining debris. We used the QIAGEN MinElute kit for purification and concentration of DNA.

\section{Omni-ATAC-Seq Library Preparation}

Saturation PCR conditions for Omni-ATAC-Seq libraries were performed using a Roche Lightcycler 480 as per the Corces et al. 2017 paper. Optimal conditions for additional amplification after pre-amplification were determined based on the number of additional PCR cycles corresponding to one-third of the maximum value, rounded up. Barcodes were added to samples using primers supplied by QB3-Berkeley using PCR as described in the Corces et al. 2017 Omni-ATAC-Seq paper. Following amplification, adapters were removed from libraries using Ampure bead purification and analyzed using a Bioanalyzer or Fragment Analyzer machine to assess library quality. Final libraries were pooled together to have relatively equal proportions based on additional qPCR quantification and size-selected using a Pippin Prep to remove fragments greater than $1.5 \mathrm{~kb}$ in size. All libraries were sequenced on one lane of each of Illumina NovaSeq SP 150PE and NovaSeq S1 150PE. Adapters used for libraries are listed in Supplementary Table 1.

\section{Omni-ATAC Sequencing Quality Control}

Adapter trimming was performed using Trim Galore, which leverages Cutadapt and FASTQC (Andrews, 2010; Kreuger, 2017; Martin, 2011). FASTQC was also performed before and after adapter trimming to confirm removal of sequencing and Tn5 adapters. Percentage of reads remaining after deduplication was estimated based on FASTQC metrics. Library size was estimated by multiplying raw read count by percentage of reads remaining after deduplication for each lane, and then summing the two lanes. Reads were aligned to the phaw_5.0 genome as well as the Parhyale chrM using Bowtie2, and percentage read mapping was determined using Bowtie2 output (Langmead and Salzberg, 2012).

\section{Omni-ATAC Peak Calling}

Aligned reads were quality-filtered with a $Q$ value of 10 in samtools and used for Genrich analysis. Output .bedgraph-like files from Genrich were reformatted using a custom Python script to be standard .bedgraph files, which were converted to bigWig files using the bedGraphToBigWig executable from the UCSC Genome Tools software bundle. Output .narrowPeak files from Genrich were converted into .bed files for ease of visualization in IGV using a custom Python script. 


\section{Assigning spatial categories to Omni-ATAC peaks}

We first assigned the nearest peak within $5 \mathrm{~kb}$ of the first $200 \mathrm{bp}$ of each gene (mRNA and ncRNA) as a promoter peak using bedtools closest. We then assigned the remaining peaks into categories based on their position relative to mRNA and ncRNA annotations. Peaks that overlapped with mRNA and ncRNA annotations were assigned as exonic or intronic regulatory elements. The remaining peaks - those which had not been classified as promoters, and which did not overlap with genes - were classified as intergenic peaks. The intergenic peaks were divided into two categories: proximal and distal intergenic peaks. Proximal peaks were those less than $10 \mathrm{~kb}$ away from the nearest gene, while distal intergenic peaks were those greater than $10 \mathrm{~kb}$ away from the nearest gene.

\section{Differential Expression and Accessibility Analyses}

We performed differential expression analysis using ImpulseDE2 and DESeq2 using standard settings. To generate the Omni-ATAC-Seq read count matrix, we used bedtools multicov, using the merged Genrich peaks as our regions of interest. For our ImpulseDE2 analyses using the Omni-ATAC-Seq data, ImpulseDE2 model fits were extracted from the ImpulseDE2 output and used to visualize model expression using a custom Python script (see Supplementary Methods). To generate the RNA-Seq read count matrix for DESeq2, we generated a gene_trans_map file for the Mikado transcriptome, as would be available for the Trinity RNA-Seq analysis pipeline, and used the built-in Trinity differential expression pipeline (align_and_estimate_abundance.pl, abundance_estimates_to_matrix.pl) with Kallisto to generate a matrix of read counts. To comply with the requirement for integer counts in DESeq2 analysis, we rounded each value to the nearest whole number.

\section{NucleoATAC Nucleosome Predictions}

Quality-filtered reads from each biological duplicate were merged and analyzed using NucleoATAC, with genomic regions set as +/-500bp windows around Genrich peaks.

\section{HINT-ATAC Transcription Factor Footprinting}

Quality-filtered reads from each biological duplicate were merged and analyzed using rgt-hint footprinting. We used the JASPAR2020 database and converted the position weight matrices from JASPAR format into a simple matrix format expected by RGT-HINT using the R package "universalmotif," and generated a ".mtf" file to store database information (see Supplementary Methods). For enrichment analyses, we used bedtools random to generate 13 million random $20 \mathrm{bp}$ sequences, as this was the average footprint size of genuine footprints detected by RGT-HINT in our data. This set of random sequences was used as background for our enrichment analyses. For cluster-specific enrichment analyses, we collated all unique transcription factor footprints from all developmental stages for each cluster (e.g. all footprints across S13, $\mathrm{S} 14$, etc. for all peaks in a given cluster) and compared enrichment levels to our randomly generated background.

\section{Minos Transposon Cloning}

Minos transposon reporter plasmids were cloned using Gibson homology-mediated cloning approaches and the NEB Gibson Assembly or NEBuilder kits. As a base plasmid, we used the pMi(ne1) plasmid, which contains the Hsp70 minimal promoter, a DsRed protein sequence, and an SV40 3'UTR sequence, as well as two Minos inverted repeats. For plasmids containing the Hsp70 minimal promoter, the insert was integrated between the EcoRV and Bglll restriction sites. For plasmids containing an endogenous promoter, the insert replaced the sequence between the EcoRV and Ncol restriction sites, thereby removing the Hsp70 minimal promoter. Cloned plasmids were Sanger sequenced to confirm a full DsRed ORF and inclusion of desired genomic sequences. 


\section{Minos Transposase Assay}

Minos transposase mRNA was generated using the ThermoFisher mMESSAGE mMACHINE T7 or T7 ULTRA kit using Notl-digested pBlueSK-MimRNA (Addgene \#102535). mRNA and concentrated DNA were mixed into a final concentration of $1 \mu \mathrm{g} / \mu \mathrm{L}$ in a solution of $0.1 \%$ phenol red in nuclease-free water. 1- and 2-cell Parhyale embryos were injected with approximately 3-5 picoliters of injection mix using a borosilicate glass capillary needle pulled using a Sutter P-80 or P-85 instrument. Embryos were raised until hatching and examined once per day from 3dpf-10dpf using a Zeiss LSM780 confocal microscope to screen for DsRed fluorescence.

\section{Data Availability}

Omni-ATAC-Seq and RNA-Seq raw reads will be available at SRA by the time of future publication. Omni-ATAC and RNA-Seq downstream analyses will be available at GEO by time of future publication. Downstream analysis files for Omni-ATAC-Seq will include BigWig files for visualizing Omni-ATAC read pileups; stage-specific and combined Genrich peaks; NucleoATAC signal, occupancy, insert, v-plots, and other miscellaneous data; HINT-ATAC transcription factor footprinting predictions and enrichment statistics; JASPAR2020 converted database and .mtf file for HINT-ATAC analyses; and differential accessibility analysis tables. Downstream analysis files for RNA-Seq will include BigWig files for visualizing RNA-Seq read pileups; Nanopore alignment sequences; individual transcriptome GFF and FASTA files; eggNOG annotations and OrthoFinder annotations of transcripts; and RNA-Seq differential expression analysis tables. In addition, the GEO database will contain the updated Mikado genome annotation and assignment of peaks based on their position relative to genes, along with classification data for peaks as concordant or discordant in expression and accessibility. Code for analysis and visualizations will be curated and deposited in a GitHub repository prior to publication.

\section{Contributions}

D.A.S. conceived of, designed, and performed experiments and analyses. D.A.S. wrote and edited the manuscript. N.H.P. provided guidance and supervised the project, and provided feedback on the manuscript.

\section{Acknowledgments}

We thank Jase Gehring and Jenna Haines for providing tips and reagents for performing and troubleshooting ATAC-Seq, and Kasia Oktaba for suggesting the technique. Jenna Haines and Shaked Afik also provided helpful programming references for performing ATAC-Seq analyses. We are grateful to Aaron Pomerantz for helping to troubleshoot and perform Nanopore sequencing, and for tips on RNA-Seq analysis. This work would have been much more challenging without fantastic sequencing support from the QB3 Biosciences Functional Genomics Lab, particularly Shana McDevitt, Karen Lundy, and Justin Choi. Thank you to the members of the Patel Lab, the Miller Lab, and the Garcia Lab for providing feedback and suggestions to the manuscript, particularly Craig Miller, Tyler Square, and Brandon Schlomann.

\section{Conflicts of Interest}

The authors declare no conflicts of interest. 


\section{References}

Andrews, S. (2010). FastQC: A Quality Control Tool for High Throughput Sequence Data.

Brogaard, K., Xi, L., Wang, J.-P., and Widom, J. (2012). A map of nucleosome positions in yeast at base-pair resolution. Nature 486, 496-501.

Browne, W.E., Price, A.L., Gerberding, M., and Patel, N.H. (2005). Stages of embryonic development in the amphipod crustacean, Parhyale hawaiensis. Genesis 42, 124-149.

Bruce, H.S., and Patel, N.H. (2020). Knockout of crustacean leg patterning genes suggests that insect wings and body walls evolved from ancient leg segments. Nat. Ecol. Evol. 4, 1703-1712.

Buenrostro, J.D., Wu, B., Chang, H.Y., and Greenleaf, W.J. (2015). ATAC-seq: A Method for Assaying Chromatin Accessibility Genome-Wide. Curr Protoc Mol Biol 109, 21.29.1-9.

Cazet, J.F., Cho, A., and Juliano, C.E. (2021). Generic injuries are sufficient to induce ectopic Wnt organizers in Hydra. ELife 10, e60562.

Corces, M.R., Trevino, A.E., Hamilton, E.G., Greenside, P.G., Sinnott-Armstrong, N.A., Vesuna, S., Satpathy, A.T., Rubin, A.J., Montine, K.S., Wu, B., et al. (2017). An improved ATAC-seq protocol reduces background and enables interrogation of frozen tissues. Nat. Methods 14, 959-962.

Costa-Silva, J., Domingues, D., and Lopes, F.M. (2017). RNA-Seq differential expression analysis: An extended review and a software tool. PLOS ONE 12, e0190152.

Crawford, K., Diaz Quiroz, J.F., Koenig, K.M., Ahuja, N., Albertin, C.B., and Rosenthal, J.J.C. (2020). Highly Efficient Knockout of a Squid Pigmentation Gene. Curr. Biol. 30, 3484-3490.e4.

Duan, Y., Zhang, W., Cheng, Y., Shi, M., and Xia, X.-Q. (2020). A systematic evaluation of bioinformatics tools for identification of long noncoding RNAs. RNA.

Emms, D.M., and Kelly, S. (2019). OrthoFinder: phylogenetic orthology inference for comparative genomics. Genome Biol. 20, 238.

Fischer, D.S., Theis, F.J., and Yosef, N. (2018). Impulse model-based differential expression analysis of time course sequencing data. Nucleic Acids Res. 46, e119-e119.

Gaspar, J.M. Genrich.

Gatzmann, F., Falckenhayn, C., Gutekunst, J., Hanna, K., Raddatz, G., Carneiro, V.C., and Lyko, F. (2018). The methylome of the marbled crayfish links gene body methylation to stable expression of poorly accessible genes. Epigenetics Chromatin 11, 57.

Gehrke, A.R., Neverett, E., Luo, Y.-J., Brandt, A., Ricci, L., Hulett, R.E., Gompers, A., Ruby, J.G., Rokhsar, D.S., Reddien, P.W., et al. (2019). Acoel genome reveals the regulatory landscape of whole-body regeneration. Science 363, eaau6173.

Gilbert, D.G. (2019). Longest protein, longest transcript or most expression, for accurate gene reconstruction of transcriptomes? BioRxiv 829184.

Giresi, P.G., Kim, J., McDaniell, R.M., Iyer, V.R., and Lieb, J.D. (2007). FAIRE (Formaldehyde-Assisted Isolation of Regulatory Elements) isolates active regulatory elements from human chromatin. Genome Res. 17, 877-885.

Haas, B.J., Papanicolaou, A., Yassour, M., Grabherr, M., Blood, P.D., Bowden, J., Couger, M.B., Eccles, D., Li, B., Lieber, M., et al. (2013). De novo transcript sequence reconstruction from RNA-seq using the Trinity platform for reference generation and analysis. Nat Protoc 8, 1494-1512.

Huerta-Cepas, J., Szklarczyk, D., Heller, D., Hernández-Plaza, A., Forslund, S.K., Cook, H., Mende, D.R., Letunic, I., Rattei, T., Jensen, L.J., et al. (2019). eggNOG 5.0: a hierarchical, functionally and phylogenetically annotated orthology resource based on 5090 organisms and 2502 viruses. Nucleic Acids Res. 47, D309-D314.

JL, and G.-S., and Shubin, N. (2015). Deep conservation of wrist and digit enhancers in fish. Proc. ....

Kao, D., Lai, A.G., Stamataki, E., Rosic, S., Konstantinides, N., Jarvis, E., Donfrancesco, A., Natalia, P.-S., Semon, M., Grillo, M., et al. (2016). The genome of the crustacean Parhyale hawaiensis, a model for animal development, regeneration, immunity and lignocellulose digestion. Elife 5, 065789.

Kissane, S., Dhandapani, V., and Orsini, L. (2021). Protocol for assay of transposase accessible chromatin sequencing in non-model species. STAR Protoc. 2, 100341.

Konstantinides, N., and Averof, M. (2014). A common cellular basis for muscle regeneration in arthropods and vertebrates. Science $343,788-791$. 
Kovaka, S., Zimin, A.V., Pertea, G.M., Razaghi, R., Salzberg, S.L., and Pertea, M. (2019). Transcriptome assembly from long-read RNA-seq alignments with StringTie2. Genome Biol. 20, 278.

Kreuger, F. (2017). TrimGalore.

Kumar, L., and E Futschik, M. (2007). Mfuzz: a software package for soft clustering of microarray data. Bioinformation 2, 5-7.

Lai, Y.-T., Deem, K.D., Borràs-Castells, F., Sambrani, N., Rudolf, H., Suryamohan, K., El-Sherif, E., Halfon, M.S., McKay, D.J., and Tomoyasu, Y. (2018). Enhancer identification and activity evaluation in the red flour beetle, Tribolium castaneum. Development 145.

Langmead, B., and Salzberg, S.L. (2012). Fast gapped-read alignment with Bowtie 2. Nat. Methods 9, 357-359.

Li, Y., Chen, C., Kaye, A.M., and Wasserman, W.W. (2015). The identification of cis-regulatory elements: A review from a machine learning perspective. Biosystems 138, 6-17.

Li, Z., Schulz, M.H., Look, T., Begemann, M., Zenke, M., and Costa, I.G. (2019). Identification of transcription factor binding sites using ATAC-seq. Genome Biol. 20, 45.

Liu, B., Li, J., and Cairns, M.J. (2014). Identifying miRNAs, targets and functions. Brief. Bioinform. 15, $1-19$.

Love, M.I., Huber, W., and Anders, S. (2014). Moderated estimation of fold change and dispersion for RNA-seq data with DESeq2. Genome Biol. 15, 550.

Ludwig, M.Z., Patel, N.H., and Kreitman, M. (1998). Functional analysis of eve stripe 2 enhancer evolution in Drosophila: rules governing conservation and change. Development 125, 949-958.

Mahony, S., and Benos, P.V. (2007). STAMP: a web tool for exploring DNA-binding motif similarities. Nucleic Acids Res. 35, W253-W258.

Martin, M. (2011). Cutadapt removes adapter sequences from high-throughput sequencing reads. EMBnetjournal Vol 17 No 1 Gener. Seq. Data Anal. - 1014806ej171200.

Martin, A., Serano, J.M., Jarvis, E., Bruce, H.S., Wang, J., Ray, S., Barker, C.A., C, O., Liam, and Patel, N.H. (2015). CRISPR/Cas9 Mutagenesis Reveals Versatile Roles of Hox Genes in Crustacean Limb Specification and Evolution. Curr Biol 26, 14-26.

Mattioli, K., Oliveros, W., Gerhardinger, C., Andergassen, D., Maass, P.G., Rinn, J.L., and Melé, M. (2020). Cis and trans effects differentially contribute to the evolution of promoters and enhancers. Genome Biol. 21, 210.

MITO, T., NAKAMURA, T., BANDO, T., OHUCHI, H., and NOJI, S. (2011). The advent of RNA interference in Entomology. Entomol. Sci. 14, 1-8.

Ofran, Y., Mysore, V., and Rost, B. (2007). Prediction of DNA-binding residues from sequence. Bioinformatics 23, i347-i353.

Paris, M., Wolff, C., Patel, N.H., and Averof, M. (2021). The Crustacean Model Parhyale hawaiensis. Preprints.

Pavlopoulos, A., and Averof, M. (2005). Establishing genetic transformation for comparative developmental studies in the crustacean Parhyale hawaiensis. Proc Natl Acad Sci USA 102, 7888-7893.

Pavlopoulos, A., Kontarakis, Z., Liubicich, D.M., Serano, J.M., Akam, M., Patel, N.H., and Averof, M. (2009). Probing the evolution of appendage specialization by Hox gene misexpression in an emerging model crustacean. Proc Natl Acad Sci USA 106, 13897-13902.

Pérez-Zamorano, B., Rosas-Madrigal, S., Lozano, O.A.M., Castillo Méndez, M., and Valverde-Garduño, V. (2017). Identification of cis-regulatory sequences reveals potential participation of lola and Deaf1 transcription factors in Anopheles gambiae innate immune response. PloS One 12, e0186435-e0186435.

Picelli, S., Björklund, Å.K., Reinius, B., Sagasser, S., Winberg, G., and Sandberg, R. (2014). Tn5 transposase and tagmentation procedures for massively-scaled sequencing projects. Genome Res 24, gr.177881.114.

Poynton, H.C., Hasenbein, S., Benoit, J.B., Sepulveda, M.S., Poelchau, M.F., Hughes, D.S.T., Murali, S.C., Chen, S., Glastad, K.M., Goodisman, M.A.D., et al. (2018). The Toxicogenome of Hyalella azteca: A Model for Sediment Ecotoxicology and Evolutionary Toxicology. Environ. Sci. Technol. 52, 6009-6022.

Quinlan, A.R., and Hall, I.M. (2010). BEDTools: a flexible suite of utilities for comparing genomic features. 
Bioinformatics 26, 841-842.

Radman-Livaja, M., and Rando, O.J. (2010). Nucleosome positioning: how is it established, and why does it matter? Dev. Biol. 339, 258-266.

Ramos, A.P., Gustafsson, O., Labert, N., Salecker, I., Nilsson, D.-E., and Averof, M. (2019). Analysis of the genetically tractable crustacean Parhyale hawaiensis reveals the organisation of a sensory system for low-resolution vision. BMC Biol. 17, 67.

Rasys, A.M., Park, S., Ball, R.E., Alcala, A.J., Lauderdale, J.D., and Menke, D.B. (2019). CRISPR-Cas9 Gene Editing in Lizards through Microinjection of Unfertilized Oocytes. Cell Rep. 28, 2288-2292.e3.

Ratnere, I., and Dubchak, I. (2009). Obtaining Comparative Genomic Data with the VISTA Family of Computational Tools. Curr. Protoc. Bioinforma. 26, 10.6.1-10.6.17.

Rehm, E.J., Hannibal, R.L., Chaw, R.C., Vargas-Vila, M.A., and Patel, N.H. (2009). Fixation and Dissection of Parhyale hawaiensis Embryos. Cold Spring Harb. Protoc. 2009, pdb.prot5127.

Robinson, M.D., McCarthy, D.J., and Smyth, G.K. (2010). edgeR: a Bioconductor package for differential expression analysis of digital gene expression data. Bioinforma. Oxf. Engl. 26, 139-140.

Rouhana, L., Weiss, J.A., Forsthoefel, D.J., Lee, H., King, R.S., Inoue, T., Shibata, N., Agata, K., and Newmark, P.A. (2013). RNA interference by feeding in vitro-synthesized double-stranded RNA to planarians: methodology and dynamics. Dev. Dyn. Off. Publ. Am. Assoc. Anat. 242, 718-730.

Schep, A.N., Buenrostro, J.D., Denny, S.K., Schwartz, K., Sherlock, G., and Greenleaf, W.J. (2015). Structured nucleosome fingerprints enable high-resolution mapping of chromatin architecture within regulatory regions. Genome Res.

Schmitt-Engel, C., Schultheis, D., Schwirz, J., Ströhlein, N., Troelenberg, N., Majumdar, U., Dao, V.A., Grossmann, D., Richter, T., Tech, M., et al. (2015). The iBeetle large-scale RNAi screen reveals gene functions for insect development and physiology. Nat. Commun. 6, 7822.

Serano, J.M., Martin, A., Liubicich, D.M., Jarvis, E., Bruce, H.S., La, K., Browne, W.E., Grimwood, J., and Patel, N.H. (2016). Comprehensive analysis of Hox gene expression in the amphipod crustacean Parhyale hawaiensis. Dev Biol 409, 297-309.

Sharma, P.P., Schwager, E.E., Giribet, G., Jockusch, E.L., and Extavour, C.G. (2013). Distal-less and dachshund pattern both plesiomorphic and apomorphic structures in chelicerates: RNA interference in the harvestman Phalangium opilio (Opiliones). Evol. Dev. 15, 228-242.

Signor, S.A., and Nuzhdin, S.V. (2018). The Evolution of Gene Expression in cis and trans. Trends Genet. 34, 532-544.

Srivastava, M., Mazza-Curll, K.L., van Wolfswinkel, J.C., and Reddien, P.W. (2014). Whole-Body Acoel Regeneration Is Controlled by Wnt and Bmp-Admp Signaling. Curr. Biol. 24, 1107-1113.

Stamataki, E., and Pavlopoulos, A. (2016). Non-insect crustacean models in developmental genetics including an encomium to Parhyale hawaiensis. Curr Opin Genet Dev 39, 149-156.

Sun, D.A., and Patel, N.H. (2019). The amphipod crustacean Parhyale hawaiensis: An emerging comparative model of arthropod development, evolution, and regeneration. WIREs Dev. Biol. 8, e355.

Venturini, L., Caim, S., Kaithakottil, G.G., Mapleson, D.L., and Swarbreck, D. (2018). Leveraging multiple transcriptome assembly methods for improved gene structure annotation. GigaScience 7.

Wang, L., and Brown, S.J. (2006). BindN: a web-based tool for efficient prediction of DNA and RNA binding sites in amino acid sequences. Nucleic Acids Res. 34, W243-W248.

Wang, Q., Gu, L., Adey, A., Radlwimmer, B., Wang, W., Hovestadt, V., Bähr, M., Wolf, S., Shendure, J., Eils, R., et al. (2013). Tagmentation-based whole-genome bisulfite sequencing. Nat Protoc 8 , 2022-2032.

Wasserman, W.W., and Sandelin, A. (2004). Applied bioinformatics for the identification of regulatory elements. Nat Rev Genet 5, 276-287.

Wetterstramd, K. DNA Sequencing Costs: Data from the NHGRI Genome Sequencing Program (GSP).

Wittkopp, P.J., Haerum, B.K., and Clark, A.G. (2004). Evolutionary changes in cis and trans gene regulation. Nature 430, 85-88.

Yan, F., Powell, D.R., Curtis, D.J., and Wong, N.C. (2020). From reads to insight: a hitchhiker's guide to ATAC-seq data analysis. Genome Biol. 21, 22. 